\title{
HEAT KERNELS ON COVERING SPACES AND TOPOLOGICAL INVARIANTS
}

\author{
JOHN LOTT
}

\section{Introduction}

It is well known that there are relationships between the heat flow, acting on differential forms on a closed oriented manifold $M$, and the topology of $M$. From Hodge theory, one can recover the Betti numbers of $M$ from the heat flow. Furthermore, Ray and Singer [42] defined an analytic torsion, a smooth invariant of acyclic flat bundles on $M$, and conjectured that it equals the classical Reidemeister torsion. This conjecture was proved to be true independently by Cheeger [7] and Müller [40]. The analytic torsion is nonzero only on odd-dimensional manifolds, and behaves in some ways as an odd-dimensional counterpart of the Euler characteristic [20].

If $M$ is not simply-connected, then there is a covering space analog of the Betti numbers. Using the heat flow on the universal cover $\widetilde{M}$, one can define the $L^{2}$-Betti numbers of $M$ [1] by taking the trace of the heat kernel not in the ordinary sense, but as an element of a certain type II von Neumann algebra. More concretely, this amounts to integrating the local trace of the heat kernel over a fundamental domain in $\widetilde{M}$. In $\S I$, we summarize this theory.

We consider the covering space analog of the analytic torsion. This $L^{2}$-analytic torsion has the same relation to the $L^{2}$-cohomology as the ordinary analytic torsion bears to de Rham cohomology. In $\S I I I$ we define the $L^{2}$-analytic torsion $\mathscr{T}_{\Gamma}(M)$, under a technical assumption which we discuss later, and prove its basic properties. We show that $\mathscr{T}_{\Gamma}(M)$ is a smooth invariant of manifolds whose $L^{2}$-Betti numbers vanish. The proof is similar to that of the analogous statement for the ordinary analytic torsion, but requires some care because of the possible slow decay of the heat kernels for large time.

In order to know if there are interesting examples of $\mathscr{T}_{\Gamma}(M)$, we compute it in the case where $M$ admits a hyperbolic metric. It is clear that $\mathscr{T}_{\Gamma}(M)$ is proportionate to the volume of the hyperbolic metric, which is

Received August 13, 1990 and, in revised form, April 26, 1991. 
a topological invariant by Mostow rigidity. The question is whether the constant of proportionality is nonzero. In $\S I V$ we show that the constant is nonzero for three-dimensional hyperbolic manifolds. Another invariant of $M$ which gives the hyperbolic volume is the simplicial volume $\|M\|$ of Gromov [25]. The relationship between $\|M\|$ and $\mathscr{T}_{\Gamma}(M)$ is not clear. Also, $\mathscr{T}_{\Gamma}(M)$ presumably equals the $L^{2}$-Reidemeister torsion defined in [6] and [31], although we do not prove this.

The technical condition needed to define $\mathscr{T}_{\Gamma}(M)$ is that the type II traces of the heat kernels approach the $L^{2}$-Betti numbers sufficiently quickly for large time. For example, a power-law decay is sufficient. It was shown by Novikov and Shubin that in addition to the $L^{2}$-Betti number $b_{p}^{(2)}(M)$, the exponent $\alpha_{p}(M)$ involved in the power-law decay of the trace on $p$-forms is a smooth invariant of $M[41]$. In $\S \mathrm{V}$ we show that $\alpha_{p}(M)$ is defined for all closed oriented topological manifolds $M$ and is a homeomorphism invariant. This is done by putting a Lipschitz structure on $M$ [47] and carrying out the heat kernel analysis on the Lipschitz manifold ([48], [28]).

In order to obtain some understanding of the invariant $\alpha_{p}(M)$, in $\S \mathrm{VI}$ we look at the case of Abelian fundamental group. One can then use Fourier analysis and the perturbation theory of operators to get some concrete results. We show that in this case $\alpha_{p}(M)$ is determined by higher order cohomology products. We give examples where $\alpha_{p}(M)$ is arbitrarily close to 0 .

In $\S$ VII we collect some information on $\alpha_{p}(M)$ in the case of locally symmetric spaces. We also consider the case of manifolds covered by Heisenberg groups and obtain upper bounds on $\alpha_{p}(M)$. For the threedimensional Heisenberg group we show that these bounds are exact. This is done by explicit calculation of the heat kernel on the diagonal of $\widetilde{M} \times \widetilde{M}$.

\section{Type II traces of heat kernels}

We give a summary of the theory of type II traces of heat kernels. For more details, see [1], [8], [14].

Let $\Gamma$ be a discrete group. There is an action $L: \Gamma \rightarrow B\left(l^{2}(\Gamma)\right)$ of $\Gamma$ on $l^{2}(\Gamma)$ induced from the action of $\Gamma$ on $\Gamma$ of left multiplication. Put

$$
\mathscr{M}=\left\{T \in B\left(l^{2}(\Gamma)\right): L(g) T=T L(g) \text { for all } g \in \Gamma\right\} .
$$

is a von Neumann algebra generated by the right multiplication operators $R(g)$. There is a finite trace $\operatorname{Tr}_{\text {II }}$ on $\mathscr{M}$, continuous in the weak 
operator topology, given by $\operatorname{Tr}_{\mathrm{II}}\left(R_{g}\right)=\delta_{g, e}$, which makes $\mathscr{M}$ into a type II $_{1}$ von Neumann algebra.

Let $M$ be a closed oriented smooth Riemannian manifold of dimension $n$ and let $\widetilde{M}$ be its universal cover. Let $\Lambda^{p}(\widetilde{M})$ denote the Hilbert space of $L^{2} p$-forms on $\widetilde{M}$. The Laplacian $\widetilde{\Delta}_{p}=d d^{*}+d^{*} d$, initially defined on smooth forms of compact support, has a unique selfadjoint extension, with domain the Sobolev space of $p$-forms $H^{2}(\widetilde{M})$ and image contained in $\Lambda^{p}(\widetilde{M})$. For $T>0$, the operator $e^{-T \widetilde{\Delta}_{p}}$ defined by the spectral theorem has a Schwartz kernel given by a smooth form on $\widetilde{M} \times \widetilde{M}$ [12].

The group $\Gamma=\pi_{1}(M)$ acts by deck transformations on $\widetilde{M}$. If $\mathscr{F}$ is a fundamental domain for this action, then $\Lambda^{p}(\widetilde{M}) \cong l^{2}(\Gamma) \otimes \Lambda^{p}(\mathscr{F})$, and the space of bounded operators on $\Lambda^{p}(\widetilde{M})$ which commute with the $\Gamma$ action can be identified with $\mathscr{M} \otimes B\left(\Lambda^{p}(\mathscr{F})\right)$. There is a (possibly infinite) trace $\operatorname{Tr}_{\Gamma}$ on $\mathscr{M} \otimes B\left(\Lambda^{p}(\mathscr{F})\right)$, constructed from $\operatorname{Tr}_{\text {II }}$ and the ordinary trace on $B\left(\Lambda^{p}(\mathscr{F})\right)$, which makes $\mathscr{M} \otimes B\left(\Lambda^{p}(\mathscr{F})\right)$ into a type $\mathrm{II}_{\infty}$ von Neumann algebra.

In particular, for $T>0, e^{-T \widetilde{\Delta}_{p}}$ is a bounded $\Gamma$-invariant operator on $\Lambda^{p}(\widetilde{M})$ and $\operatorname{Tr}_{\Gamma} e^{-T \widetilde{\Delta}_{p}}$ is finite. More explicitly,

$$
\operatorname{Tr}_{\Gamma} e^{-T \widetilde{\Delta}_{p}}=\int_{\mathscr{F}} \operatorname{tr}\left(e^{-T \tilde{\Delta}_{p}}(x, x)\right) d \operatorname{vol}(x),
$$

where tr denotes the (finite-dimensional) trace on $\operatorname{End}\left(\Lambda^{p}(x)\right)$. The $L^{2}$ Betti numbers are given by

$$
b_{p}^{(2)}(M)=\lim _{T \rightarrow \infty} \operatorname{Tr}_{\Gamma} e^{-T \widetilde{\Delta}_{p}} .
$$

\section{III. $L^{2}$-analytic torsion}

First, recall the definition of the usual analytic torsion [42]. Let $\Lambda^{p}(M)$ denote the Hilbert space of $L^{2} p$-forms on $M$ and put $\Lambda^{*}(M)=$ $\bigoplus \Lambda^{p}(M)$. Let $\Delta$ denote the Laplacian on $\Lambda^{*}(M)$, a densely defined selfadjoint operator. Let $\Delta^{\prime}$ denote the Laplacian acting on $(\operatorname{Ker} \Delta)^{\perp}$. Let $F: \Lambda^{*}(M) \rightarrow \Lambda^{*}(M)$ be the operator which is multiplication by $p$ on $\Lambda^{p}(M)$.

Definition 1. We set

$$
\mathscr{T}=\left.\frac{d}{d s}\right|_{s=0} \frac{1}{\Gamma(s)} \int_{0}^{\infty} T^{s-1} \operatorname{Tr}(-1)^{F} F e^{-T \Delta^{\prime}} d T .
$$


The integral, which exists for $\operatorname{Re} s>n / 2$, is extended analytically to a meromorphic function in $s$, so that one is differentiating a holomorphic function at $s=0$.

We now form the $L^{2}$-analog of $\mathscr{T}$. Let $\tilde{\Delta}^{\prime}$ denote the Laplacian acting on $(\operatorname{Ker} \widetilde{\Delta})^{\perp}$.

\section{Definition 2.}

$$
\begin{aligned}
\mathscr{T}_{\Gamma}= & \left.\frac{d}{d s}\right|_{s=0} \frac{1}{\Gamma(s)} \int_{0}^{\varepsilon} T^{s-1} \operatorname{Tr}_{\Gamma}(-1)^{F} F e^{-T \widetilde{\Delta}^{\prime}} d T \\
& +\int_{\varepsilon}^{\infty} T^{-1} \operatorname{Tr}_{\Gamma}(-1)^{F} F e^{-T \widetilde{\Delta}^{\prime}} d T
\end{aligned}
$$

Note 3. 1. We are assuming that for all $p$, as $T \rightarrow \infty, \operatorname{Tr}_{\Gamma} e^{-T \widetilde{\Delta}_{p}^{\prime}}$ is $O\left(T^{-\alpha_{p} / 2}\right)$ for some $\alpha_{p}>0$. Then the second integral in (5) makes sense. This asymptotic condition is independent of the Riemannian metric (see $\S \mathrm{V})$. We do not know if it is always fulfilled.

2. We will see below that the first integral in (5) can be extended analytically in $s$ from $\operatorname{Re} s>n / 2$.

3. $\mathscr{T}_{\Gamma}$ is independent of the choice of the positive number $\varepsilon$ in the definition.

4. We use zeta-function regularization on $[0, \varepsilon]$. This is in order to ensure that $\mathscr{T}_{\Gamma}$ has a simple dependence on the Riemannian metric.

5. We do not extend the zeta-function regularization to large time. This is because $\operatorname{Tr}_{\Gamma}(-1)^{F} F e^{-T \widetilde{\Delta}^{\prime}}$ may have a slow decay for large $T$, which could mean that the integrand of an expression analogous to (4) would not be integrable for any value of $s$.

6. One could make the same definition for a normal cover of $M$ with group of deck transformations $\Gamma$.

In order to study the small- $T$ behavior of $\operatorname{Tr}_{\Gamma} e^{-T \widetilde{\Delta}_{p}}$, one can use a parametrix $\widetilde{P}$ on $\widetilde{M}$ which is pulled back from a parametric $P$ on $M$. It follows that $\operatorname{Tr}_{\Gamma} e^{-T \widetilde{\Delta}_{p}}$ and $\operatorname{Tr} e^{-T \Delta_{p}}$ have the same small- $T$ asymptotics. More precisely, we have

Lemma 4. Let $d$ be the length of the shortest closed geodesic on $M$ which is in a nontrivial free homotopy class. Put $N=[n / 4]+1$. Then for any integer $k \geq 0$ and any $\varepsilon>0,\left|\operatorname{Tr}_{\Gamma} \widetilde{\Delta}_{p}^{k} e^{-T \widetilde{\Delta}_{p}}-\operatorname{Tr} \Delta_{p}^{k} e^{-T \Delta_{p}}\right|$ is $O\left(T^{-2 k-4 N+(1 / 2)} e^{-(d-\varepsilon)^{2} / 4 T}\right)$ as $T \rightarrow 0$.

Proof. Let $\varepsilon$ be arbitrarily small. For $x, y \in \widetilde{M}$, put $R=d(x, y)-\varepsilon$. If $d(x, y)>\varepsilon$, the finite propagation speed method of $[11, \S 1]$ gives 


$$
\left|\widetilde{\Delta}_{p}^{k} e^{-T \widetilde{\Delta}_{p}}(x, y)\right| \leq \text { const. } \sum_{i=0}^{2 N} \int_{R}^{\infty}\left|\hat{f}^{(2 k+2 i)}(s)\right| d s,
$$

where $\hat{f}(s)=\pi^{1 / 2} T^{-1 / 2} e^{-s^{2} / 4 T}$. (We are not concerned with the geometric dependence of the constants, which is the main point of [11].) Now

$$
\hat{f}^{(2 j)}(s)=T^{-(j+1 / 2)} P_{j}\left(\frac{s^{2}}{T}\right) e^{-s^{2} / 4 T}
$$

for some polynomial $P_{j}$ of degree $j$, and so

$$
\begin{aligned}
\int_{R}^{\infty} & \left|\hat{f}^{(2 j)}(s)\right| d s \\
& \leq \text { const. } \int_{R}^{\infty} T^{-(j+1 / 2)}\left[1+\left(\frac{s^{2}}{T}\right)^{j}\right] e^{-s^{2} / 4 T} d s \\
& \leq \text { const. } \int_{0}^{\infty} T^{-(j+1 / 2)}\left[1+\left(\frac{(R+x)^{2}}{T}\right)^{j}\right] e^{-\left(R^{2}+2 R x\right) / 4 T} d x \\
& \leq \text { const } . T^{-(j+1 / 2)}\left[1+\left(\frac{(R+T / R)^{2}}{T}\right)^{j}\right] e^{-R^{2} / 4 T} \frac{T}{R} \\
& \leq \text { const } . T^{-j}\left(\frac{R^{2}}{T}\right)^{-1 / 2}\left[\left(\frac{R^{2}}{T}\right)^{-j}+\left(\frac{R^{2}}{T}\right)^{j}\right] e^{-R^{2} / 4 T} .
\end{aligned}
$$

Thus

$$
\begin{aligned}
\left|\tilde{\Delta}_{p}^{k} e^{-T \widetilde{\Delta}_{p}}(x, y)\right| \leq \text { const } . & \left(\frac{R^{2}}{T}\right)^{-1 / 2}\left[R^{-2 k}+R^{-2 k-4 N}\right. \\
& \left.+R^{2 k} T^{-2 k}+R^{2 k+4 N} T^{-2 k-4 N}\right] e^{-R^{2} / 4 T} .
\end{aligned}
$$

Let $a: \Gamma \rightarrow \operatorname{Diff}(\widetilde{M})$ denote the action of $\Gamma$ on $\widetilde{M}$ by deck transformations. Put

$$
F(x, t, T)=\sum_{g}\left(\tilde{\Delta}_{p}^{k} e^{-T \widetilde{\Delta}_{p}} a(g)^{*}\right)(x, y) \quad \text { for } x, y \in \widetilde{M} .
$$

To see that the sum in (10) converges absolutely, put $N(r)=\#\left(\{a(g) y\}_{g \in \Gamma}\right.$ $\left.\cap \overline{B_{r}(x)}\right\}$. If the sectional curvatures of $M$ are $\geq-K^{2}$, then $N(r) \leq$ const. $e^{(n-1) K r}$ [37]. Thus (9) gives 


$$
\begin{aligned}
& \sum_{g}\left|\left(\widetilde{\Delta}_{p}^{k} e^{-T \widetilde{\Delta}_{p}} a(g)^{*}\right)(x, y)\right| \\
& \leq \text { const } .(T)\left[1+\int_{\varepsilon}^{\infty}(r-\varepsilon)^{2 k+4 N-1} e^{-(r-\varepsilon)^{2} / 4 T} d(N(r)-1)\right] \\
& \text { = const. } .(T)\left[1+\int_{0}^{\infty} R^{2 k+4 N-1} e^{-R^{2} / 4 T} d(N(R+\varepsilon)-1)\right] \\
& \text { = const } .(T)\left[1+\int_{0}^{\infty}\left[-(2 k+4 N-1) R^{2 k+4 N-2}+\frac{R^{2 k+4 N}}{2 T}\right]\right. \\
& \left.\times e^{-R^{2} / 4 T}(N(R+\varepsilon)-1) d R\right] \\
& \leq \text { const } .(T)\left[1+\int_{0}^{\infty}\left[-(2 k+4 N-1) R^{2 k+4 N-2}+\frac{R^{2 k+4 N}}{2 T}\right]\right. \\
& \left.\times e^{-R^{2} / 4 T} e^{(n-1) K R} d R\right]<\infty
\end{aligned}
$$

We claim

$$
F(x, y, T)=\left(\Delta_{p}^{k} e^{-T \Delta_{p}}\right)(\pi(x), \pi(y))
$$

To see this, by construction we have $\left(a\left(g_{1}\right)^{*} F a\left(g_{2}\right)^{*}\right)(T)=F(T)$ for all $g_{1}, g_{2} \in \Gamma$, and so

$$
F(T)=\pi^{*} G(T)
$$

for some smooth form $G$ on $M \times M$. Also

$$
\left(\frac{\partial}{\partial T}+\widetilde{\Delta}_{p}\right) F(T)=\left(\frac{\partial}{\partial T}+\widetilde{\Delta}_{p}\right) \pi^{*} G(T)=\pi^{*}\left(\frac{\partial}{\partial T}+\Delta_{p}\right) G(T),
$$

which implies

$$
\left(\frac{\partial}{\partial T}+\Delta_{p}\right) G(T)=0
$$

As distributions on $\widetilde{M} \times \widetilde{M}$,

$$
\lim _{T \rightarrow 0} F(T)=\lim _{T \rightarrow 0} \sum_{g} \tilde{\Delta}_{p}^{k} e^{-T \tilde{\Delta}_{p}} a(g)^{*}=\widetilde{\Delta}_{p}^{k}=\pi^{*} \Delta_{p}^{k},
$$

so $\lim _{T \rightarrow 0} G(T)=\Delta_{p}^{k}$. By the uniqueness of solutions to the heat equation on $M$, we have

$$
G(T)=\Delta_{p}^{k} e^{-T \Delta_{p}}
$$


Now

$$
\begin{gathered}
\left(\Delta_{p}^{k} e^{-T \Delta_{p}}\right)(\pi(x), \pi(x))-\left(\tilde{\Delta}_{p}^{k} e^{-T \tilde{\Delta}_{p}}\right)(x, x) \\
=\sum_{g \neq e}\left(\tilde{\Delta}_{p}^{k} e^{-T \widetilde{\Delta}_{p}}\left(a(g)^{*}\right)(x, x) .\right.
\end{gathered}
$$

For any $\Lambda>0$, let us divide this sum into the contribution of $\{g$ : $d(x, a(g) x) \leq \Lambda+\varepsilon\}$ and the contribution of $\{g: d(x, a(g) x)>\Lambda+\varepsilon\}$. The second contribution is bounded by

$$
\begin{aligned}
& \int_{\Lambda}^{\infty} \text { const. }\left(\frac{R^{2}}{T}\right)^{-1 / 2}\left[R^{-2 k}+R^{-2 k-4 N}+R^{2 k} T^{-2 k}+R^{2 k+4 N} T^{-2 k-4 N}\right] \\
& \times e^{-R^{2} / 4 T} d(N(R+\varepsilon)-N(\Lambda+\varepsilon)) \\
& \leq \int_{\Lambda}^{\infty} \text { const . } \mid \frac{d}{d R}\left\{( \frac { R ^ { 2 } } { T } ) ^ { - 1 / 2 } \left[R^{-2 k}+R^{-2 k-4 N}+R^{2 k} T^{-2 k}\right.\right. \\
& \left.\left.+R^{2 k+4 N} T^{-2 k-4 N}\right] e^{-R^{2} / 4 T}\right\} \mid e^{(n-1) K R} d R
\end{aligned}
$$

$$
\begin{aligned}
& \leq \int_{\Lambda}^{\infty} \text { const. } T^{-1 / 2}\left(1+\frac{R^{2}}{T}\right)\left[R^{-2 k-2}+R^{-2 k-4 N-2}\right. \\
& \left.\quad+R^{2 k-2} T^{-2 k}+R^{2 k+4 N-2} T^{-2 k-4 N}\right] e^{-R^{2} / 4 T} e^{(n-1) K R} d R \\
& \leq \text { const } e^{-\Lambda^{2} / 4 T} e^{(n-1) K \Lambda} \int_{0}^{\infty} T^{-1 / 2}\left(1+\frac{(\Lambda+x)^{2}}{T}\right) \\
& \quad \times\left[(\Lambda+x)^{-2 k-2}+(\Lambda+x)^{-2 k-4 N-2}+(\Lambda+x)^{2 k-2} T^{-2 k}\right. \\
& \left.+(\Lambda+x)^{2 k+4 N-2} T^{-2 k-4 N}\right] e^{-2 \Lambda x / 4 T} e^{(n-1) K x} d x .
\end{aligned}
$$

By taking $\Lambda$ large enough, we can ensure that the result is $o\left(e^{-100 d^{2} / T}\right)$ as $T \rightarrow 0$ uniformly with respect to $x \in \widetilde{M}$.

We are left with the contribution of the finite set $\{g: d(x, a(g) x) \leq$ $\Lambda+\varepsilon\}$. By (9), this is $O\left(T^{-2 k-4 N+(1 / 2)} e^{-(d-\varepsilon)^{2} / T}\right)$ as $T \rightarrow 0$, uniformly with respect to $x$. Integrating (18) over a fundamental domain thus gives the lemma.

\section{Corollary 5.}

$$
\mathscr{T}_{\Gamma}-\mathscr{T}=\int_{0}^{\infty} T^{-1}\left(\operatorname{Tr}_{\Gamma}(-1)^{F} F e^{-T \tilde{\Delta}}-\operatorname{Tr}(-1)^{F} F e^{-T \Delta}\right.
$$

$$
\left.-\theta(T-\varepsilon)\left(c_{\Gamma}-c\right)\right) d T-(\ln \varepsilon+\gamma)\left(c_{\Gamma}-c\right)
$$


where $\theta$ is the Heaviside step function, $\varepsilon$ is an arbitrary positive number, $\gamma$ is the Euler-Mascheroni constant, $c_{\Gamma}=\sum(-1)^{p} p b_{p}^{(2)}$, and $c=$ $\sum(-1)^{p} p b_{p}$. (The point is that there is no need for zeta-function regularization in $\mathscr{T}_{\Gamma}-\mathscr{T}$.)

Proof. We have

$$
\begin{aligned}
\mathscr{T}_{\Gamma}-\mathscr{T}= & \left.\frac{d}{d s}\right|_{s=0} \frac{1}{\Gamma(s)} \int_{0}^{\varepsilon} T^{s-1}\left[\operatorname{Tr}_{\Gamma}(-1)^{F} F e^{-T \tilde{\Delta}}\right. \\
& \left.\quad-c_{\Gamma}-\operatorname{Tr}(-1)^{F} F e^{-T \Delta}+c\right] \\
& +\int_{\varepsilon}^{\infty} T^{-1}\left[\operatorname{Tr}_{\Gamma}(-1)^{F} F e^{-T \tilde{\Delta}}-c_{\Gamma}-\operatorname{Tr}(-1)^{F} F e^{-T \Delta}+c\right] d T .
\end{aligned}
$$

By Lemma 4, $\int_{0}^{\varepsilon} T^{s-1}\left(\operatorname{Tr}_{\Gamma}(-1)^{F} F e^{-T \tilde{\Delta}}-\operatorname{Tr}(-1)^{F} F e^{-T \Delta}\right) d T$ is holomorphic in $s$, and so

$$
\begin{aligned}
\mathscr{T}_{\Gamma}-\mathscr{T}= & \int_{0}^{\varepsilon} T^{-1}\left[\operatorname{Tr}_{\Gamma}(-1)^{F} F e^{-T \widetilde{\Delta}}-\operatorname{Tr}(-1)^{F} F e^{-T \Delta}\right] d T \\
(22) \quad & -\left.\frac{d}{d s}\right|_{s=0} \frac{1}{s \Gamma(s)}\left(c_{\Gamma}-c\right) \varepsilon^{s} \\
& +\int_{\varepsilon}^{\infty} T^{-1}\left[\operatorname{Tr}_{\Gamma}(-1)^{F} F e^{-T \widetilde{\Delta}}-\operatorname{Tr}(-1)^{F} F e^{-T \Delta}-\left(c_{\Gamma}-c\right)\right] d T,
\end{aligned}
$$

from which the corollary follows.

Proposition 6. If $M$ is even dimensional, then $\mathscr{T}_{\Gamma}(M)=0$.

Proof. This follows from the Hodge duality, as in the usual case [42].

Proposition 7. Suppose that $M$ is odd dimensional. If $g(u)=g_{0} u+$ $g_{1}(1-u)$ is a one-parameter family of Riemannian metrics, let $*(u)$ denote the Hodge duality operator for the metric $g(u)$, and put $V=\left(\left.\frac{d}{d u}\right|_{u=0} * *^{-1}\right.$. Then

$$
\left.\frac{d}{d u}\right|_{u=0} \mathscr{T}_{\Gamma}(u)=\left.\operatorname{Tr}_{\Gamma}(-1)^{F+1} V\right|_{\operatorname{Ker} \widetilde{\Delta}}
$$

Proof. We will think of $V$ as an operator on both $\Lambda^{*}(M)$ and $\Lambda^{*}(\widetilde{M})$. By the known formula for $\frac{d}{d u} \mathscr{T}(u)$ [42], it is enough to show

$$
\left.\frac{d}{d u}\right|_{u=0}\left(\mathscr{T}_{\Gamma}(u)-\mathscr{T}(u)\right)=\left.\operatorname{Tr}_{\Gamma}(-1)^{F+1} V\right|_{\operatorname{Ker} \widetilde{\Delta}}-\left.\operatorname{Tr}(-1)^{F+1} V\right|_{\operatorname{Ker} \Delta}
$$


Define

$$
\begin{aligned}
\left(\mathscr{T}_{\Gamma}-\mathscr{T}\right)(u, \Lambda)= & \int_{0}^{\Lambda} T^{-1}\left[\operatorname{Tr}_{\Gamma}(-1)^{F} F e^{-T \widetilde{\Delta}}-\operatorname{Tr}(-1)^{F} F e^{-T \Delta}\right. \\
& -(\ln \varepsilon+\gamma)\left(c_{\Gamma}-c\right) .
\end{aligned}
$$

Then

$$
\left(\mathscr{T}_{\Gamma}-\mathscr{T}\right)(u)=\lim _{\Lambda \rightarrow \infty}\left(\mathscr{T}_{\Gamma}-\mathscr{T}\right)(u, \Lambda)
$$

We will show that

$$
\frac{d}{d u}\left(\mathscr{T}_{\Gamma}-\mathscr{T}\right)(u, \Lambda)=\operatorname{Tr}_{\Gamma}(-1)^{F+1} V e^{-\Lambda \widetilde{\Delta}}-\operatorname{Tr}(-1)^{F+1} V e^{-\Lambda \Delta}
$$

so that

$$
\left(\mathscr{T}_{\Gamma}-\mathscr{T}\right)(u, \Lambda)=\left(\mathscr{T}_{\Gamma}-\mathscr{T}\right)(0, \Lambda)
$$

$$
+\int_{0}^{u}\left[\operatorname{Tr}_{\Gamma}(-1)^{F+1} V e^{-\Lambda \tilde{\Delta}}-\operatorname{Tr}(-1)^{F+1} V e^{-\Lambda \Delta}\right](v) d v
$$

Using the assumed large-time decay of $\operatorname{Tr}_{\Gamma} e^{-T \widetilde{\Delta^{\prime}}}$, it will follow that

$$
\left(\mathscr{T}_{\Gamma}-\mathscr{T}\right)(u)=\left(\mathscr{T}_{\Gamma}-\mathscr{T}\right)(0)
$$

$$
+\int_{0}^{u}\left[\left.\operatorname{Tr}_{\Gamma}(-1)^{F+1} V\right|_{\operatorname{Ker} \widetilde{\Delta}}-\left.\operatorname{Tr}(-1)^{F+1} V\right|_{\operatorname{Ker} \Delta}\right](v) d v
$$

Lemma 8. $B(u, T) \equiv \operatorname{Tr}_{\Gamma}(-1)^{F} F e^{-T \widetilde{\Delta}}-\operatorname{Tr}(-1)^{F} F e^{-T \Delta}$ is differentiable in $u$, with

$$
\frac{d B}{d u}=T \frac{d}{d T}\left(\operatorname{Tr}_{\Gamma}(-1)^{F+1} V e^{-T \widetilde{\Delta}}-\operatorname{Tr}(-1)^{F+1} V e^{-T \Delta}\right)
$$

Proof. By Duhamel's principle, we have

$$
\begin{aligned}
& \frac{1}{u}\left[\operatorname{Tr}_{\Gamma}(-1)^{F} F e^{-(T-\varepsilon) \tilde{\Delta}(u)} e^{-\varepsilon \widetilde{\Delta}(0)}-\operatorname{Tr}_{\Gamma}(-1)^{F} F e^{-\tilde{\Delta}(u)} e^{-(T-\varepsilon) \widetilde{\Delta}(0)}\right] \\
& \quad=-\int_{\varepsilon}^{T-\varepsilon} \operatorname{Tr}_{\Gamma}(-1)^{F} F e^{-\tilde{s}(u)} u^{-1}(\widetilde{\Delta}(u)-\widetilde{\Delta}(0)) e^{-(T-s) \tilde{\Delta}(0)} d s .
\end{aligned}
$$

Because $\left\|\left(u^{-1}(\widetilde{\Delta}(u)-\widetilde{\Delta}(0))-\left.\frac{d}{d u}\right|_{u=0} \widetilde{\Delta}(u)\right) e^{-\varepsilon / \widetilde{\Delta}(0)}\right\|$ is $O(u)$ as $u \rightarrow 0$, the 
limit as $u \rightarrow 0$ of (31) exists, and we can write

$$
\begin{aligned}
\left.\frac{d}{d u}\right|_{u=0}\left[\operatorname{Tr}_{\Gamma}(-1)^{F}\right. & \left.F e^{-(T-\varepsilon) \widetilde{\Delta}(u)} e^{-\varepsilon \widetilde{\Delta}(0)}-\operatorname{Tr}_{\Gamma}(-1)^{F} F e^{-\varepsilon \widetilde{\Delta}(u)} e^{-(T-\varepsilon) \tilde{\Delta}(0)}\right] \\
& =-\left.\int_{\varepsilon}^{T-\varepsilon} \operatorname{Tr}_{\Gamma}(-1)^{F} F e^{-s \widetilde{\Delta}(0)} \frac{d \widetilde{\Delta}}{d u}\right|_{u=0} e^{-(T-s) \tilde{\Delta}(0)} d s \\
& =-\left.(T-2 \varepsilon) \operatorname{Tr}_{\Gamma}(-1)^{F} F \frac{d \widetilde{\Delta}}{d u}\right|_{u=0} e^{-T \widetilde{\Delta}(0)} .
\end{aligned}
$$

As in [9], we have

$$
\begin{aligned}
\left.\frac{d}{d u}\right|_{u=0} \operatorname{Tr}_{\Gamma}(-1)^{F} F e^{-T \widetilde{\Delta}(u)}= & \left.\frac{d}{d u}\right|_{u=0} \operatorname{Tr}_{\Gamma}(-1)^{F} F e^{-\varepsilon \widetilde{\Delta}(u)} e^{-(T-\varepsilon) \widetilde{\Delta}(u)} \\
= & \operatorname{Tr}_{\Gamma}(-1)^{F} F\left(\left.\frac{d}{d u}\right|_{u=0} e^{-\varepsilon \widetilde{\Delta}(u)}\right) e^{-(T-\varepsilon) \widetilde{\Delta}(0)} \\
& +\left.\operatorname{Tr}_{\Gamma}(-1)^{F} F e^{-\varepsilon \widetilde{\Delta}(0)} \frac{d}{d u}\right|_{u=0} e^{-(T-\varepsilon) \widetilde{\Delta}(u)},
\end{aligned}
$$

and therefore

$$
\lim _{\varepsilon \rightarrow 0} \operatorname{Tr}_{\Gamma}(-1)^{F} F\left(\left.\frac{d}{d u}\right|_{u=0} e^{-\varepsilon \tilde{\Delta}(u)}\right) e^{-(T-\varepsilon) \widetilde{\Delta}(0)}=0 .
$$

It follows that

$$
\left.\frac{d}{d u}\right|_{u=0} \operatorname{Tr}_{\Gamma}(-1)^{F} F e^{-T \tilde{\Delta}(u)}=-\left.T \operatorname{Tr}_{\Gamma}(-1)^{F} F \frac{d \tilde{\Delta}}{d u}\right|_{u=0} e^{-T \tilde{\Delta}(0)}
$$

From this point on, the arguments are the same as for the ordinary analytic torsion [7]. q.e.d.

Using the method of proof of Lemma 4 in the case $k=1$, we have that $\frac{d}{d T}\left[\operatorname{Tr}_{\Gamma}(-1)^{F} V e^{-T \widetilde{\Delta}}-\operatorname{Tr}(-1)^{F} V e^{-T \Delta}\right]$ is uniformly bounded for $0 \leq$ $T \leq \Lambda$ and $0 \leq u \leq 1$. Then (27) follows. In order to take the limit $\Lambda \rightarrow \infty$ in (28), because $V$ is uniformly bounded in $v$, it suffices to know that $\operatorname{Tr}_{\Gamma} e^{-\widetilde{\Delta}_{p}}-\operatorname{Tr}_{\Gamma}$ (projection onto $\operatorname{Ker} \widetilde{\Delta}_{p}$ ) is $O\left(\Lambda^{-\alpha_{p} / 2}\right.$ ) for some $\alpha_{p}$, uniformly in $v \in[0, u]$. By assumption, this is true for any fixed $v \in[0, u]$. Lemma 25 and the proof of Proposition 32 will show that it is true uniformly on $[0, u]$.

Corollary 9. If $M$ is $L^{2}$-acyclic, then $T_{\Gamma}$ is a smooth invariant of $M$.

Note 10. Some examples of odd-dimensional $L^{2}$-acyclic manifolds are given by nonpositively-curved locally symmetric spaces, manifolds whose 
sectional curvature is sufficiently closely pinched around -1 [17], and $K(\pi, 1)$ manifolds with $\pi$ amenable [10].

\section{Proposition 11.}

$$
\mathscr{T}_{\Gamma}\left(M_{1} \times M_{2}\right)=\chi\left(M_{1}\right) \mathscr{T}_{\Gamma}\left(M_{2}\right)+\chi\left(M_{2}\right) \mathscr{T}_{\Gamma}\left(M_{1}\right) .
$$

Proof. We have

$$
\widetilde{\Delta}\left(\widetilde{M_{1}} \times \widetilde{M_{2}}\right)=\widetilde{\Delta}\left(\widetilde{M_{1}}\right) \otimes I+I \otimes \widetilde{\Delta}\left(\widetilde{M_{2}}\right)
$$

and so

$$
\begin{aligned}
\operatorname{Tr}_{\Gamma}(-1)^{F} F e^{-T \widetilde{\Delta}\left(\widetilde{M_{1}} \times \widetilde{M}_{2}\right)}= & \operatorname{Tr}_{\Gamma}(-1)^{F_{1}+F_{2}}\left(F_{1}+F_{2}\right) e^{-T \widetilde{\Delta}\left(\widetilde{M_{1}}\right)} \otimes e^{-T \widetilde{\Delta}\left(\widetilde{M_{2}}\right)} \\
= & \operatorname{Tr}_{\Gamma}(-1)^{F_{1}} F_{1} e^{-T \widetilde{\Delta}\left(\widetilde{M}_{1}\right)} \operatorname{Tr}_{\Gamma}(-1)^{F_{2}} e^{-T \widetilde{\Delta}\left(\widetilde{M_{2}}\right)} \\
& +\operatorname{Tr}_{\Gamma}(-1)^{F_{1}} e^{-T \widetilde{\Delta}\left(\widetilde{M_{1}}\right)} \operatorname{Tr}_{\Gamma}(-1)^{F_{2}} F_{2} e^{-T \widetilde{\Delta}\left(\widetilde{M_{2}}\right)} .
\end{aligned}
$$

Now $\operatorname{Tr}_{\Gamma}(-1)^{F_{2}} e^{-T \widetilde{\Delta}\left(\widetilde{M}_{2}\right)}$ is independent of $T$ and equals $\sum_{s}(-1)^{s} b_{s}^{(2)}\left(M_{2}\right)$ [8], which equals $\chi\left(M_{2}\right)$ by Atiyah's $L^{2}$ index theorem [1]. Thus

$$
\begin{aligned}
\operatorname{Tr}_{\Gamma}(-1)^{F} F e^{-T \widetilde{\Delta}\left(\widetilde{M_{1}} \times \widetilde{M}_{2}\right)}= & \chi\left(M_{1}\right) \operatorname{Tr}_{\Gamma}(-1)^{F_{2}} F_{2} e^{-T \widetilde{\Delta}\left(\widetilde{M_{2}}\right)} \\
& +\chi\left(M_{2}\right) \operatorname{Tr}_{\Gamma}(-1)^{F_{1}} F_{1} e^{-T \widetilde{\Delta}\left(\widetilde{M_{1}}\right)}
\end{aligned}
$$

Subtracting the $T \rightarrow \infty$ limit of (39) gives

$$
\begin{aligned}
\operatorname{Tr}_{\Gamma}(-1)^{F} F e^{-T \widetilde{\Delta^{\prime}}\left(\widetilde{M_{1}} \times \widetilde{M_{2}}\right)}= & \chi\left(M_{1}\right) \operatorname{Tr}_{\Gamma}(-1)^{F_{2}} F_{2} e^{-T \widetilde{\Delta}^{\prime}\left(\widetilde{M_{2}}\right)} \\
& +\chi\left(M_{2}\right) \operatorname{Tr}_{\Gamma}(-1)^{F_{1}} F_{1} e^{-T \widetilde{\Delta^{\prime}}\left(\widetilde{M_{1}}\right)}
\end{aligned}
$$

from which the proposition then follows.

Proposition 12. $\mathscr{T}_{\Gamma}$ is multiplicative under finite coverings.

Proof. This follows from the multiplicativity of $\operatorname{Tr}_{\Gamma} e^{-T \widetilde{\Delta}_{p}}$.

Lemma 13. For any $\alpha>0$,

$$
\left.\frac{d}{d s}\right|_{s=0} \frac{1}{\Gamma(s)} \int_{0}^{\varepsilon} T^{s-1} T^{-\alpha / 2} d T+\int_{\varepsilon}^{\infty} T^{-1} T^{-\alpha / 2} d T=0 .
$$

Proof. The left-hand side equals

$$
\left.\frac{d}{d s}\right|_{s=0} \frac{1}{\Gamma(s)} \frac{\varepsilon^{s-(\alpha / 2)}}{s-(\alpha / 2)}+\frac{\varepsilon^{-(\alpha / 2)}}{\alpha / 2}
$$

Because $1 / \Gamma(s)=s+O\left(s^{2}\right)$, we thus obtain the lemma.

Corollary 14. If $M$ is finitely covered by $S^{1} \times N$ for some closed oriented $N$, then $\mathscr{T}_{\Gamma}(M)=0$. 
Proof. By Lemma 13 and the explicit heat kernel on $\mathbf{R}$, we have $\mathscr{T}_{\Gamma}\left(S^{1}\right)=0$. The corollary then follows from Propositions 11 and 12 .

\section{Torsion of hyperbolic manifolds}

If $M$ is a locally homogeneous space, and $h(x, y)$ is the Schwartz kernel of the projection onto $\operatorname{Ker} \widetilde{\Delta}$, then

$$
\begin{aligned}
\frac{\mathscr{T}_{\Gamma}}{\operatorname{Vol}(M)}= & \left.\frac{d}{d s}\right|_{s=0} \frac{1}{\Gamma(s)} \int_{0}^{\varepsilon} T^{s-1} \operatorname{tr}(-1)^{F} F\left[e^{-T \widetilde{\Delta}}(x, x)-h(x, x)\right] d T \\
& +\int_{\varepsilon}^{\infty} T^{-1} \operatorname{tr}(-1)^{F} F\left(e^{-T \tilde{\Delta}}(x, x)-h(x, x)\right) d T
\end{aligned}
$$

for any $x \in \widetilde{M}$. Thus if $M$ is a manifold which is $L^{2}$-acyclic and admits a locally homogeneous metric, then $\mathscr{T}_{\Gamma}(M)$, a smooth invariant of $M$, is proportionate to the volume of the locally homogeneous space (after the curvature has been appropriately normalized). The constant of proportionality depends only on the geometry of $\widetilde{M}$, and the question is whether it is nonzero. If $M$ admits the structure of an irreducible locally symmetric space of nonpositive curvature, then it follows from [39, Proposition 2.1] that $\mathscr{T}_{\Gamma}(M)$ vanishes unless $\widetilde{M}=\mathrm{SO}(p, q) / \mathrm{S}(\mathrm{O}(p) \times \mathrm{O}(q))$ with $p$ and $q$ odd, or $\widetilde{M}=\mathrm{SL}(3, \mathbf{R}) / \mathrm{SO}(3)$.

We will compute $\mathscr{T}_{\Gamma}(M)$ in the special case

$$
\widetilde{M}=H^{d}=\mathrm{SO}(d, 1) / \mathrm{S}(\mathrm{O}(d) \times \mathrm{O}(1))
$$

with $d$ odd. Note that $H^{d}$ is $L^{2}$-acyclic for $d$ odd [16]. Using the Hodge decomposition, we can rewrite $\mathscr{T}_{\Gamma} / \operatorname{Vol}(M)$ in this case as

$$
\begin{aligned}
\frac{\mathscr{T}_{\Gamma}}{\operatorname{Vol}(M)}= & \left.\frac{d}{d s}\right|_{s=0} \frac{1}{\Gamma(s)} \int_{0}^{\varepsilon} T^{s-1}\left[\operatorname{tr}(-1)^{F+1} e^{-T \tilde{\Delta}}(x, x)\right] d T \\
& +\int_{\varepsilon}^{\infty} T^{-1}\left[\operatorname{tr}(-1)^{F+1} e^{-T \tilde{\Delta}}(x, x)\right] d T
\end{aligned}
$$

where $\widetilde{\Delta}$ is now the Laplacian acting on coclosed forms. Thus to compute $T_{\Gamma}$ it suffices to know

$$
F_{j}(T)=\operatorname{tr} e^{-T \widetilde{\Delta}_{j}}(x, x),
$$

the local trace of the heat kernel on coclosed $j$-forms. 
Proposition 15. For $H^{d}$ with $d$ odd,

$$
\begin{aligned}
F_{j}(T)= & (4 \pi)^{-d / 2} \frac{1}{\Gamma(d / 2)}\left(\begin{array}{c}
d-1 \\
j
\end{array}\right) \\
& \times \int_{-\infty}^{\infty}\left(\exp \left(-T\left(\nu^{2}+\left(\frac{d-1}{2}-j\right)^{2}\right)\right)\right) \\
& \times\left(\prod_{i=0}^{(d-1) / 2}\left(\nu^{2}+i^{2}\right)\right)\left(\nu^{2}+\left(\frac{d-1}{2}-j\right)^{2}\right)^{-1} d \nu .
\end{aligned}
$$

This is a consequence of the more general results of Miatello [36]. For our special case, let us put $d=2 n+1$ and define the groups $G=\operatorname{SO}(d, 1)$, $K=\mathrm{SO}(d)$, and $M=\mathrm{SO}(d-1)$. (For this proof, $M$ will always refer to this group, for which the notation seems to be standard.) Then $H^{d}=G / K$, and a homogeneous vector bundle over $H^{d}$ is given by a finite-dimensional representation $\tau$ of $K$. Because $d$ is odd, there is no discrete series contribution to the heat kernel, and only the induced series contribution, which is specified by the restriction of $\tau$ to $M$. In our case the coclosed $j$-forms correspond to the representation $\sigma_{j}$ of $M$ on $\Lambda^{j}\left(\mathbf{R}^{d-1}\right)$ [19]. As $G$ is of rank one, the corresponding induced series is labelled by a real number $\nu$, and the representation of the Casimir of $g$ on the $\nu$ representation is $\nu^{2}+(n-j)^{2}$ [19]. Thus

$$
F_{j}(T)=(4 \pi)^{-d / 2} \frac{1}{\Gamma(d / 2)}\left(\begin{array}{c}
2 n \\
j
\end{array}\right) \int_{-\infty}^{\infty}\left(\exp \left(-T\left(\nu^{2}+(n-j)^{2}\right)\right)\right) P_{\sigma_{j}}(\nu) d \nu
$$

where $P_{\sigma_{j}}(\nu) d \nu$ is the Plancherel measure. In the notation of [35], the highest weight of the representation $\sigma_{j}$ is $\Lambda_{\sigma_{j}}=\sum_{i=2}^{n+1} s_{i} \varepsilon_{i}$, with $s_{2}=$ $\cdots=s_{j+1}=1, s_{j+2}=\cdots=s_{n+1}=0$. Then

$$
P_{\sigma_{j}}(\nu)=\alpha\left(\prod_{k=0}^{n}\left(\beta^{2} \nu^{2}+k^{2}\right)\right)\left(\beta^{2} \nu^{2}+(n-j)^{2}\right)^{-1}
$$

for some constants $\alpha$ and $\beta$ [35]. The constants $\alpha$ and $\beta$ are in principle given in [35], but because of the many normalizations we prefer to compute them directly. This can be done using the small-time heat kernel asymptotics. First, we have

$$
F_{j}(T) \sim\left(\begin{array}{c}
2 n \\
j
\end{array}\right) \alpha \beta^{2 n}(4 \pi T)^{-d / 2}=\left(\begin{array}{c}
2 n \\
j
\end{array}\right)(4 \pi T)^{-d / 2},
$$


so $\alpha \beta^{2 n}=1$. Second, because $\beta$ is independent of $j$, we can use the expansion

$$
\begin{aligned}
F_{0}(T) & \sim(4 \pi T)^{-d / 2} e^{-T n^{2}}\left(1+\frac{1}{3} n(n-1) \beta^{-2} T\right) \\
& \sim(4 \pi T)^{-d / 2}\left(1+\frac{R}{6} T\right) .
\end{aligned}
$$

With constant sectional curvature $-1, R=-d(d-1)$ and so $\beta=1$. q.e.d.

It is now straightforward to compute $\mathscr{T}_{\Gamma}$ for hyperbolic 3-manifolds.

Proposition 16. If $M^{3}$ is hyperbolic, then $\mathscr{T}_{\Gamma}\left(M^{3}\right) / \operatorname{Vol}\left(M^{3}\right)=$ $-1 /(3 \pi)$.

Proof. Putting $d=3$, we get

$$
F_{0}(T)=(4 \pi T)^{-3 / 2} e^{-T}, \quad F_{1}(T)=\frac{2(1+2 T)}{(4 \pi T)^{-3 / 2}}, \quad F_{2}(T)=F_{0}(T) .
$$

(This agrees with [50].) By Lemma $13, F_{1}(T)$ does not contribute to $\mathscr{T}_{\Gamma}$. We are left with

$$
\begin{aligned}
\mathscr{T}_{\Gamma}= & -\left.2 \frac{d}{d s}\right|_{s=0} \frac{1}{\Gamma(s)} \int_{0}^{\varepsilon} T^{s-1}(4 \pi T)^{-3 / 2} e^{-T} d T \\
& -2 \int_{\varepsilon}^{\infty} T^{-1}(4 \pi T)^{-3 / 2} e^{-T} d T \\
= & -\left.2 \frac{d}{d s}\right|_{s=0} \frac{1}{\Gamma(s)} \int_{0}^{\infty} T^{s-1}(4 \pi T)^{-3 / 2} e^{-T} d T \\
= & -\left.2 \frac{d}{d s}\right|_{s=0}(4 \pi)^{-3 / 2} \frac{\Gamma(s-3 / 2)}{\Gamma(s)} \\
= & -2(4 \pi)^{-3 / 2} \Gamma\left(-\frac{3}{2}\right)=-\frac{1}{3 \pi} . \quad \text { q.e.d. }
\end{aligned}
$$

Analogously, one can compute $\mathscr{T}_{\Gamma}$ for any odd-dimensional hyperbolic space. We find

$$
\frac{\mathscr{T}_{\Gamma}\left(M^{5}\right)}{\operatorname{Vol}\left(M^{5}\right)}=-\frac{3}{2 \pi^{2}}, \quad \frac{\mathscr{T}_{\Gamma}\left(M^{7}\right)}{\operatorname{Vol}\left(M^{7}\right)}=\frac{11}{2 \pi^{3}} .
$$

We do not know if $\mathscr{T}_{\Gamma}\left(M^{2 n+1}\right) / \operatorname{Vol}\left(M^{2 n+1}\right)$ is nonzero for all $n>0$.

Note 17. If we assume that the $L^{2}$ analytic torsion $\mathscr{T}_{\Gamma}(M)$ is the same as the $L^{2}$ Reidemeister torsion $\mathscr{T}_{\Gamma}^{c}(M)$, then it follows that $\mathscr{T}_{\Gamma}(M)$ vanishes for any Seifert 3-manifold with infinite $\pi_{1}$. One way to see this is to note that the Seifert 3-manifolds all admit a locally homogeneous metric [44], and so it suffices to check the statement for one example of a Seifert 3-manifold for each of the five relevant homogeneous geometries. 
Suppose that $F \rightarrow M \rightarrow B$ is a fiber bundle and let $\cdots \rightarrow \pi_{1}(F) \stackrel{i}{\rightarrow}$ $\pi_{1}(M) \stackrel{j}{\rightarrow} \pi_{1}(B)$ be the exact homotopy sequence. Let $F^{\prime}$ be the normal covering of $F$ with covering group $\Gamma=i\left(\pi_{1}(F)\right)$. Suppose that $F$ is $\Gamma$ acyclic. Then $M$ is $L^{2}$-acyclic and the $L^{2}$ Reidemeister torsion of $M$ is $T_{\Gamma}^{\mathcal{C}}(F) \chi(B)$ [31]. It now suffices to take a circle bundle as the example of a Seifert 3-manifold for each of the five relevant homogeneous geometries, as $\mathscr{T}_{\Gamma}^{c}\left(S^{1}\right)=0$. The same argument shows that a 3 -manifold based on the Sol geometry has vanishing $L^{2}$ torsion.

Remark 18. For three-dimensional hyperbolic manifolds, one can think of the eta invariant as the imaginary part of a complex function whose real part is the volume ([52] and references therein). Also, when one computes the regularized determinant of a first-order selfadjoint elliptic operator, one can think of the phase of the determinant as given by the eta function of the operator [30]. We wish to point out that these two facts are related.

Consider the partition function $Z$ of a quantum field theory whose action is the Chern-Simons invariant of a $U(1)$ gauge theory on a closed 3-manifold $M$. Formally,

$$
Z=-\ln \int e^{-\int_{M} A \wedge d A} \mathscr{D} A,
$$

where $A \in \Lambda^{1}(M)$. This expression is formally computed [46] as

$$
Z=\frac{1}{2} \ln \operatorname{det}(T)-\ln \operatorname{det} \Delta_{0},
$$

where $T$ is the operator on $\Lambda^{1}(M) \oplus \Lambda^{3}(M)$ given by

$$
T(\omega)= \begin{cases}(d *-* d)(\omega) & \text { if } \omega \in \Lambda^{1}(M), \\ -(d *-* d)(\omega) & \text { if } \omega \in \Lambda^{3}(M) .\end{cases}
$$

We can interpret $\operatorname{Re} \ln \operatorname{det}(T)$ as

$$
R e \ln \operatorname{det}(T)=\frac{1}{2} \ln \operatorname{det}\left(T^{2}\right)=\frac{1}{2}\left(\ln \operatorname{det}\left(\Delta_{1}\right)+\ln \operatorname{det}\left(\Delta_{3}\right)\right),
$$

SO

$$
\operatorname{Re} Z=-\frac{3}{4} \ln \operatorname{det} \Delta_{0}+\frac{1}{4} \ln \operatorname{det} \Delta_{1}=-\frac{1}{4} \mathscr{T} .
$$

The imaginary part of $\ln \operatorname{det}(T)$ can be interpreted as meaning $\frac{1}{2} i \pi / \eta(M)$. Thus we interpret $Z$ as

$$
Z=-\frac{1}{4} \mathscr{T}+\frac{i \pi}{4} \eta(M)
$$


If we were to carry out the above reasoning in an $L^{2}$ setting, we would obtain

$$
Z^{(2)}=-\frac{1}{4} \mathscr{T}_{\Gamma}+\frac{i \pi}{4} \eta_{\Gamma}(M)=-\frac{1}{4} \mathscr{T}_{\Gamma}+\frac{i \pi}{4} \eta(M)+\frac{i \pi}{4}\left(\eta_{\Gamma}(M)-\eta(M)\right) .
$$

Furthermore, $\eta_{\Gamma}(M)-\eta(M)$ is metric-independent [9].

For a hyperbolic 3-manifold, from Proposition 16 we have

$$
-\frac{1}{4} \mathscr{T}_{\Gamma}+\frac{i \pi}{4} \eta(T)=\frac{\operatorname{Vol}(M)}{12 \pi}+\frac{i \pi}{4} \eta(M) .
$$

This is exactly the combination of $\operatorname{Vol}(M)$ and $\eta(M)$ which enters in [52]. More precisely, [52] defines an analytic function $f(u)$ on the deformation space of a finite volume hyperbolic 3-manifold $N$ with cusps. The deformed 3-manifold $N_{u}$ is incomplete. When the deformation is such that $N_{u}$ can be completed to a closed smooth hyperbolic 3-manifold $M_{u}$ by adding geodesic loops, then $f(u)=\frac{12}{\pi}\left[\operatorname{Vol}\left(M_{u}\right) /(12 \pi)+\frac{i \pi}{4} \eta\left(M_{u}\right)\right]+$ correction terms.

This numerology suggests that it may be possible to define $\mathscr{T}_{\Gamma}\left(N_{u}\right)$ and $\eta_{\Gamma}\left(N_{u}\right)$ for the incomplete manifolds $N_{u}$ so that the analytic function $f(u)$ equals $\frac{12}{\pi}\left[-\frac{1}{4} \mathscr{T}_{\Gamma}+\frac{i \pi}{4} \eta_{\Gamma}\left(N_{u}\right)\right]$, up to a constant. (Note that if $M$ is a closed hyperbolic manifold, then $\eta_{\Gamma}(M)=0$ because of the orientationreversing Cartan involution on $\widetilde{M}$.)

\section{Large-time decay of heat kernels}

Definition 19. The $p$ th Novikov-Shubin invariant of $M$ is

$$
\alpha_{p}(M)=\sup \left\{\beta_{p}: \operatorname{Tr}_{\Gamma} e^{-T \widetilde{\Delta}_{p}^{\prime}} \text { is } O\left(T^{-\beta_{p} / 2}\right) \text { as } T \rightarrow \infty\right\}
$$

It was shown in [41], [18] that $\alpha_{p}$ is a smooth invariant of $M$. (We use a different normalization than [41], [18].)

We will show that $\alpha_{p}$ is defined for all closed oriented topological manifolds $M$ and is a homeomorphism invariant. $\alpha_{p}(M)$ is defined by putting a Lipschitz structure on $M$ and forming the corresponding heat kernel [48], [28]. The invariance will follow along the lines of [41], [18].

A Lipschitz structure on $M$ is a maximal collection of coordinate charts $\left\{V_{\alpha}, \varphi_{\alpha}\right\}$ such that the transition functions $\varphi_{\beta} \circ \varphi_{\alpha}^{-1}: V_{\alpha} \rightarrow V_{\beta}$ are Lipschitz functions on the domains $V_{\alpha} \subset \mathbf{R}^{n}$. If $\operatorname{dim} M \neq 4$, a topological 
manifold $M$ admits a Lipschitz structure which is unique up to a homeomorphism isotopic to the identity [47]. $\widetilde{M}$ inherits a Lipschitz structure from $M$.

Because the partial derivatives of a Lipschitz function are in $L^{\infty}$, there is a well-defined measure class on $M$ (and $\widetilde{M}$ ), and it makes sense to talk of the space $\Lambda_{\text {loc }}^{*}(\widetilde{M})$ of locally $L^{2}$ differential forms. It also makes sense to talk of a Riemannian metric $g$ on $M$, given in a coordinate chart by a matrix of measurable functions which is uniformly bounded above and below almost everywhere. We will also denote by $g$ the lift of the Riemannian metric to $\widetilde{M}$. There is an associated volume form $d$ vol and Hodge duality operator $*: \Lambda_{\mathrm{loc}}^{*}(\widetilde{M}) \rightarrow \Lambda_{\mathrm{loc}}^{*}(\widetilde{M})$. Define the Hilbert space of $L^{2}$ differential forms

$$
\Lambda^{*}(\widetilde{M})=\left\{\omega \in \Lambda_{\mathrm{loc}}^{*}(\widetilde{M}): \int_{\widetilde{M}} \omega \wedge * \omega<\infty\right\}
$$

As a topological vector space, this is independent of $g$.

Let $\Lambda_{d}^{*}(\widetilde{M})$ be the dense linear subspace of $\Lambda^{*}(\widetilde{M})$ given by

$$
\Lambda_{d}^{*}(\widetilde{M})=\left\{\omega \in \Lambda^{*}(\widetilde{M}): \text { the current } d \omega \text { is in } \Lambda^{*}(\widetilde{M})\right\}
$$

That is, $\omega \in \Lambda_{d}^{*}(\widetilde{M})$ iff $\exists \eta \in \Lambda^{*}(\widetilde{M})$ such that $\int_{\widetilde{M}} \omega \wedge d \varphi= \pm \int_{\widetilde{M}} \eta \wedge \varphi$ for all $\varphi$ which are smooth forms of compact support with respect to some coordinate chart $\left(V_{\alpha}, \varphi_{\alpha}\right)$ of $\widetilde{M}$. Then the operator $d: \Lambda^{*}(\widetilde{M}) \rightarrow \Lambda^{*}(\widetilde{M})$ is densely-defined on $\Lambda_{d}^{*}(\widetilde{M})$, closed, and $d^{2}=0$.

It follows from Lemma 4.3 of [28] that $d+d^{*}$ is a densely-defined selfadjoint operator on $\Lambda^{*}(\widetilde{M})$, and $\Lambda^{*}(\widetilde{M})=\left(\operatorname{Ker} d \cap \operatorname{Ker} d^{*}\right) \oplus \overline{\operatorname{Im} d} \oplus$ $\overline{\operatorname{Im} d^{*}}$. Although it is not strictly necessary, let us say explicitly what $d^{*}$ is.

Definition 20. For $\omega \in \Lambda^{*}(\widetilde{M})$, let $\|\omega\|$ denote the norm of $\omega$, an $L^{2}$ function on $\widetilde{M}$ defined by $\omega \wedge * \omega=\|\omega\|^{2} d$ vol.

Lemma 21. There is a sequence of functions $f_{n} \in \Lambda_{d}^{*}(\widetilde{M})$ such that

1. $f_{n}$ has compact support,

2. $\left\|f_{n}\right\|_{\infty}$ is uniformly bounded in $n$,

3. for almost all $x \in \widetilde{M}$, as $n \rightarrow \infty, f_{n}(x) \rightarrow 1$,

4. as $n \rightarrow \infty,\left\|d f_{n}\right\|_{\infty} \rightarrow 0$.

Proof. Take a finite covering $\left\{U_{\alpha}\right\}$ of $M$ by coordinate charts and let $\left\{\tilde{U}_{\alpha}, \tilde{\varphi}_{\alpha}\right\}$ be the lifts of $\left\{U_{\alpha}, \varphi_{\alpha}\right\}$ to $\widetilde{M}$. Let $\tilde{d}_{\alpha}$ be the Euclidean 
distance on $\tilde{\varphi}_{\alpha}^{-1}\left(\widetilde{U}_{\alpha}\right) \subset \widetilde{M}$. For $x, y \in \widetilde{M}$, define [32]

$d(x, y)=\inf _{n, \vec{x}}\left\{\sum_{i=1}^{n-1} \tilde{d}_{\alpha_{i}}\left(x_{i}, x_{i+1}\right): x_{1}=x, x_{n}=y, x_{i}\right.$ and $x_{i+1}$

are both in a coordinate neighborhood

$$
\left.\tilde{\varphi}_{\alpha_{i}}^{-1}\left(\widetilde{U}_{\alpha_{i}}\right) \text { for all } 1 \leq i \leq n-1\right\} \text {. }
$$

Then one can check that $d_{x}(\cdot) \equiv d(x, \cdot)$ is a Lipschitz function on $\widetilde{M}$, $\left\|d d_{x}\right\|$ is in $L^{\infty}$, and $d_{x}(y)>0$ if $x \neq y$. Because $(\widetilde{M}, d)$ is a complete length space in the sense of [23], $d_{x}: \widetilde{M} \rightarrow \mathbf{R}$ is proper.

Fix $x \in \widetilde{M}$. Let $\varphi$ be a smooth bump function on $\mathbf{R}$ with $\operatorname{supp} \varphi \subset$ $[-1,1], \varphi(x)=1$ for $-\frac{1}{2} \leq x \leq \frac{1}{2}$, and put $f_{n}(y)=\varphi\left(\frac{1}{n} d_{x}(y)\right)$. Then

$$
\left(d f_{n}\right)(y)=\frac{1}{n} \varphi^{\prime}\left(\frac{1}{n} d_{x}(y)\right)\left(d d_{x}\right)(y),
$$

and the lemma follows.

Note 22. If one wants to consider a more general class of noncompact manifolds, let $X$ be a topological manifold with a uniform Lipschitz structure, meaning that there is a maximal atlas of coordinate charts $\left\{\varphi_{\alpha}, V_{\alpha}\right\}$ such that the transition functions $\varphi_{\beta} \circ \varphi_{\alpha}^{-1}$ are $K$-Lipschitz for all $\alpha$ and $\beta$, for some constant $K$. With a fixed covering of $X$ by coordinate charts, define the metric $d(x, y)$ as above. Let us assume that $X$ is complete with respect to $d$; this is independent of the covering chosen. Given a Riemannian metric $g$ on $X$ which is uniformly bounded above and below with respect to the Euclidean norms on the charts almost everywhere, one can prove the lemma in the same way. Alternatively, one can use the metric on $X$ defined intrinsically by $g$ ([13], [29]).

Proposition 23. $\operatorname{Dom}\left(d^{*}\right)=* \operatorname{Dom}(d)$ and $d^{*}=* d *(-1)^{n F+n+1}$.

Proof. Given the cutoff functions $\left\{f_{n}\right\}$, the proof is the same as in the smooth case. Let us first show $* \operatorname{Dom}(d) \subset \operatorname{Dom}\left(d^{*}\right)$. Suppose $* \eta \in$ $\operatorname{Dom}(d)$. We have to show that for all $\omega \in \operatorname{Dom}(d),\langle\omega, * d * \eta\rangle=$ $\pm\langle d \omega, \eta\rangle$. Let $\left\{\tau^{i}\right\}$ be a local measurable orthonormal basis of sections of $T^{*} \widetilde{M}$. Then

$$
\langle\omega, * d * \eta\rangle= \pm \int \omega \wedge d * \eta= \pm \int \sum_{I} \omega_{I}(* d * \eta)_{I} d \text { vol },
$$

with an obvious notation. Because $\omega$ and $* d * n$ are $L^{2}$ forms, 
$\sum_{I} \omega_{I}(* d * n)_{I} d$ vol is integrable. By Dominated Convergence, we have

$$
\begin{aligned}
\langle\omega, * d * \eta\rangle & =\lim _{n \rightarrow \infty} \pm \int f_{n} \omega \wedge d * \eta \\
& =\lim _{n \rightarrow \infty}\left( \pm \int d f_{n} \wedge \omega \wedge * \eta \pm \int f_{n} d \omega \wedge * \eta\right) .
\end{aligned}
$$

Since $\omega$ and $* \eta$ are $L^{2}$ forms, the first term has limit 0 . For the second term, because $d \omega$ and $* \eta$ are $L^{2}$ forms, the Dominated Convergence again implies that the limit is

$$
\pm \int d \omega \wedge * \eta= \pm\langle d \omega, \eta\rangle
$$

To show $\operatorname{Dom}\left(d^{*}\right) \subset * \operatorname{Dom}(d)$, suppose that there exist $L^{2}$ forms $\eta$ and $\sigma$ such that $\langle d \omega, \eta\rangle=\langle\omega, \sigma\rangle$ for all $\omega \in \operatorname{Dom}(d)$. We have to show $* \eta \in \operatorname{Dom}(d)$. Now

$$
\int d \omega \wedge * \eta=\langle d \omega, \eta\rangle=\langle\omega, \sigma\rangle=\int \omega \wedge * \sigma .
$$

Letting $\omega$ range over smooth forms with compact support with respect to a coordinate chart, we see that the distributional exterior derivative $d * \eta$ is $L^{2}$ and is given by $\pm * \sigma$. Thus $* \eta \in \operatorname{Dom}(d)$ and $\sigma=d^{*} \eta= \pm * d * \eta$. q.e.d.

Using the spectral theorem, one can define $e^{-T \widetilde{\Delta}}=e^{-T\left(d+d^{*}\right)^{2}}$, a bounded selfadjoint operator on $\Lambda^{*}(\widetilde{M})$. In order to show that $e^{-T \tilde{\Delta}}$ is in the von Neumann algebra $\mathscr{M} \otimes B\left(\Lambda^{*}(\mathscr{F})\right)$, or more generally that $f(\widetilde{\Delta})$ is in $\mathscr{M} \otimes B\left(\Lambda^{*}(\mathscr{F})\right)$ for any bounded Borel function $f:(0, \infty) \rightarrow \mathbf{R}$, it is enough to show that $d+d^{*}$ is affiliated with $\mathscr{M} \otimes B\left(\Lambda^{*}(\mathscr{F})\right)$ in the sense that

$$
B\left(d+d^{*}\right)=\left(d+d^{*}\right) B \quad \text { for all } B \in\left(\mathscr{M} \otimes B\left(\Lambda^{*}(\mathscr{F})\right)\right)^{\prime}[14] .
$$

Lemma 24. $d+d^{*}$ is affiliated with $\mathscr{M} \otimes B\left(\Lambda^{*}(\mathscr{F})\right)$.

Proof. If $g \in \pi_{1}(M)$, then clearly $a(g)^{*}$ preserves $\operatorname{Dom}\left(d+d^{*}\right)$ and

$$
a(g)^{*}\left(d+d^{*}\right)=\left(d+d^{*}\right) a(g)^{*} .
$$

Let $B_{i}$ be a sequence of finite sums $B_{i}=\sum c_{i j} a\left(g_{j}\right)^{*}$. If $\left\{B_{i}\right\}$ converges to an operator $B$ in the weak operator topology, then for all $x, y \in$ $\operatorname{Dom}\left(d+d^{*}\right)$,

$$
\begin{aligned}
\left\langle\left(d+d^{*}\right) x, B y\right\rangle & =\lim _{i}\left\langle\left(d+d^{*}\right) x, B_{i} y\right\rangle \\
& =\lim _{i}\left\langle x, B_{i}\left(d+d^{*}\right) y\right\rangle=\left\langle x, B\left(d+d^{*}\right) y\right\rangle .
\end{aligned}
$$


Thus $B y \in \operatorname{Dom}\left(d+d^{*}\right)^{*}=\operatorname{Dom}\left(d+d^{*}\right)$ and $\left(d+d^{*}\right) B y=$ $B\left(d+d^{*}\right) y$. Because $\left(\mathscr{M} \otimes B\left(\Lambda^{*}(\mathscr{F})\right)\right)^{\prime}$ is the von Neumann algebra generated by $\left\{a(g)^{*}\right\}_{g \in \pi_{1}(M)}$, the lemma follows.

Lemma 25 [28]. Let $g$ and $g^{\prime}$ be Lipschitz Riemann metrics on $M$, and let $d+d^{*}(g)$ and $d+d^{*}\left(g^{\prime}\right)$ be the corresponding selfadjoint operators on $\Lambda^{*}(\widetilde{M}, g)$ and $\Lambda^{*}\left(\widetilde{M}, g^{\prime}\right)$. Then there is an invertible bounded operator $B: \Lambda^{*}(\widetilde{M}, g) \rightarrow \Lambda^{*}\left(\widetilde{M}, g^{\prime}\right)$ such that

$$
B^{*} d B=d \quad \text { and } B^{*} d^{*}\left(g^{\prime}\right) B=d^{*}(g) \text {. }
$$

Proof. We refer to [28]. For completeness, let us give the operator $B$. Let $I$ and $J$ be the identity operators $I: \Lambda^{*}(\widetilde{M}, g) \rightarrow \Lambda^{*}\left(\widetilde{M}, g^{\prime}\right)$ and $J: \Lambda^{*}\left(\widetilde{M}, g^{\prime}\right) \rightarrow \Lambda^{*}(\widetilde{M}, g)$. Let $p, q$, and $r$ be the orthogonal projections on $\overline{\operatorname{Im} d^{*}}, \overline{\operatorname{Im} d}$, and $\operatorname{Ker} d \cap \operatorname{Ker} d^{*}$ in $\Lambda^{*}(\widetilde{M}, g)$, and similarly for $p^{\prime}, q^{\prime}$, and $r^{\prime}$. Then $B=p^{\prime} I p+q^{\prime} J^{*} q+r^{\prime} I r$.

Definition 26. $E_{p}(\lambda, g)=\chi_{[0, \lambda]}\left(\widetilde{\Delta}_{p}(g)\right)$ and $N_{p}(\lambda, g)=\operatorname{Tr}_{\Gamma} E_{p}(\lambda, g)$.

Lemma 27. $N_{p}\left(\|B\|^{4} \lambda, g\right) \geq N_{p}\left(\lambda, g^{\prime}\right)$.

Proof. We will not assume that $B$ is invertible for the proof. For simplicity we will drop the index $p$. By the generalization of Glazman's lemma ([18], [45]) we have

$$
\begin{array}{r}
N(\lambda, g)=\sup \left\{\operatorname{Tr}_{\Gamma} P: P \text { is a projection in } \mathscr{M} \otimes B\left(\Lambda^{*}(\mathscr{F})\right),\right. \\
\operatorname{Im} P \subset \operatorname{Dom}(\widetilde{\Delta}(g)) \text { and } P(\tilde{\Delta}(g)-\lambda) P \leq 0\} .
\end{array}
$$

Let $P$ be the projection onto the preimage under $B$ of $\operatorname{Im} \chi_{[0, \lambda]}\left(\tilde{\Delta}\left(g^{\prime}\right)\right)$. By Lemma 25, $\operatorname{Im} P \subset \operatorname{Dom}(\widetilde{\Delta}(g))$. Thus

$$
\begin{aligned}
\langle x, & \left.P\left(\widetilde{\Delta}(g)-\|B\|^{4} \lambda\right) P x\right\rangle=|d P x|^{2}+\left|d^{*}(g) P x\right|^{2}-\lambda\|B\|^{4}|P x|^{2} \\
& =\left|B^{*} d B P x\right|^{2}+\left|B^{*} d^{*}\left(g^{\prime}\right) B P x\right|^{2}-\lambda\|B\|^{4}|P x|^{2} \\
& \leq\|B\|^{2}\left(|d B P x|^{2}+\left|d^{*}\left(g^{\prime}\right) B P x\right|^{2}\right)-\lambda\|B\|^{4}|P x|^{2} \\
& \leq\|B\|^{2} \lambda|B P x|^{2}-\lambda\|B\|^{4}|P x|^{2} \leq 0 .
\end{aligned}
$$

From $\operatorname{Tr}_{\Gamma} P \geq N\left(\lambda, g^{\prime}\right)$ [10] hence follows the lemma.

Corollary 28. $\quad N_{p}\left(\|B\|^{4} \lambda, g\right) \geq N_{p}\left(\lambda, g^{\prime}\right) \geq N_{p}\left(\left\|B^{-1}\right\|^{-4} \lambda, g\right)$.

Corollary 29. The condition $0 \in \operatorname{Spec}\left(\widetilde{\Delta}_{p}^{\prime}(g)\right)$ is independent of the metric $g$.

Proof. $0 \notin \operatorname{Spec}\left(\tilde{\Delta}_{p}^{\prime}(g)\right)$ iff $N_{p}(\lambda, g)$ is locally constant near 0 .

Note 30. In the case $p=0$, we know that $0 \in \operatorname{Spec}\left(\tilde{\Delta}_{0}(g)\right)$ iff $\pi_{1}(M)$ is amenable [4]. The easy part of Brooks' theorem extends to the case of general $p$. Namely, if $H^{p}(M, \mathbf{R}) \neq 0$ and $\pi_{1}(M)$ is amenable, then 
$0 \in \operatorname{Spec}\left(\widetilde{\Delta}_{p}(g)\right)$. This can be seen by taking a harmonic $p$-form $\omega$ on $M$, a sequence of functions $f_{E}$ on $\widetilde{M}$ as defined in [4], and computing the Rayleigh quotients for the test forms $f_{E} \pi^{*}(\omega)$. If in addition $b_{p}^{(2)}(M)=$ 0 , then $0 \in \operatorname{Spec}\left(\widetilde{\Delta}_{p}^{\prime}(g)\right)$.

Definition 31. If $g$ is a Lipschitz Riemannian metric on $M$, then

$$
\alpha_{p}(M, g)=\sup \left\{\beta_{p}: \operatorname{Tr}_{\Gamma} e^{-T \widetilde{\Delta}_{p}^{\prime}} \text { is } O\left(T^{-\beta_{p} / 2}\right) \text { as } T \rightarrow \infty\right\}
$$

(It follows from the finite speed of propagation on Lipschitz manifolds, as proved in [29], that $\operatorname{Tr}_{\Gamma} e^{-\widetilde{\Delta_{p}^{\prime}}}$ is finite for all $T>0$.)

Proposition 32 [18], [41]). $\quad \alpha_{p}(M, g) \leq \alpha_{p}\left(M, g^{\prime}\right)$.

Proof. We will not assume that $B$ is invertible for the proof. We have

$$
\begin{aligned}
\operatorname{Tr}_{\Gamma} e^{-\widetilde{\Delta^{\prime}}(g)} & =\int_{0}^{\infty} e^{-T \lambda} d\left(N(\lambda, g)-N\left(0^{+}, g\right)\right) \\
& =T \int_{0}^{\infty} e^{-T \lambda}\left(N(\lambda, g)-N\left(0^{+}, g\right)\right) d \lambda \\
& =\|B\|^{4} T \int_{0}^{\infty} e^{-T\|B\|^{4} \lambda}\left(N\left(\|B\|^{4} \lambda, g\right)-N\left(0^{+}, g\right)\right) d \lambda \\
& \geq T\|B\|^{4} \int_{0}^{\infty} e^{-T\|B\|^{4} \lambda}\left(N\left(\lambda, g^{\prime}\right)-N\left(0^{+}, g^{\prime}\right)\right) d \lambda \\
& =\operatorname{Tr}_{\Gamma} e^{-\|B\|^{4} T \widetilde{\Delta}^{\prime}\left(g^{\prime}\right)} .
\end{aligned}
$$

Corollary 33. $\alpha_{p}(M, g)=\alpha_{p}\left(M, g^{\prime}\right)$.

Note 34. Proposition 32 holds in great generality. All that one needs is two Hilbert spaces $K_{0}$ and $K_{1}$, closely densely defined operators $d_{0}$ and $d_{1}$ with $d_{0}^{2}=d_{1}^{2}=0$, and maps $I: K_{0} \rightarrow K_{1}$ and $J: K_{1} \rightarrow K_{0}$ such that $d_{0}=J d_{1} I$. In particular, if $M$ is smooth, take $K_{0}$ to be the $L^{2}$ simplicial cochains of a triangulation of $\widetilde{M}$ pulled back from a smooth triangulation of $M, K_{1}$ to be the Sobolev space of differential forms on $\widetilde{M}$ as used in [15], $d_{0}$ and $d_{1}$ to be the coboundary maps, $I$ to be the Whitney map of [15], and $J$ to be the de Rham map. Then it follows that the combinatorial invariant $\alpha_{p}^{c}(M)$ satisfies $\alpha_{p}^{c}(M) \leq \alpha_{p}(M, g)$.

Definition 35. If $M$ is a closed oriented topological manifold with $\operatorname{dim} M \neq 4$, put a Lipschitz structure $\mathscr{L}$ on $M$ [47] and define

$$
\alpha_{p}(M, \mathscr{L}) \equiv \alpha_{p}(M, \mathscr{L}, g)
$$

for some Lipschitz Riemannian metric $g$. If $\operatorname{dim} M=4$, put a Lipschitz structure $\mathscr{L}$ on $M \times S^{6}$ and define

$$
\alpha_{p}(M, \mathscr{L}) \equiv \alpha_{\rho}\left(M \times S^{6}, \mathscr{L}, g\right)
$$

for some Lipschitz Riemannian metric $g$ on $M \times S^{6}$. 
Proposition 36. $\alpha_{p}(M, \mathscr{L})$ is independent of $\mathscr{L}$ and is a homeomorphism invariant of $M$.

Proof. First, suppose $\operatorname{dim} M \neq 4$. If $\left(M, \mathscr{L}_{1}\right)$ and $\left(M, \mathscr{L}_{2}\right)$ are two Lipschitz structures on $M$, let $\sigma:\left(M, \mathscr{L}_{1}\right) \rightarrow\left(M, \mathscr{L}_{2}\right)$ be a Lipschitz homeomorphism, which exists by [47]. Let $g_{1}$ and $g_{2}$ be Lipschitz Riemannian metrics for $\left(M, \mathscr{L}_{1}\right)$ and $\left(M, \mathscr{L}_{2}\right)$ respectively. Then

$$
\begin{aligned}
\alpha_{p}\left(M, \mathscr{L}_{1}\right) & =\alpha_{p}\left(M, \mathscr{L}_{1}, g_{1}\right)=\alpha_{p}\left(M, \mathscr{L}_{1}, \sigma^{*} g_{2}\right) \\
& =\alpha_{p}\left(M, \mathscr{L}_{2}, g_{2}\right)=\alpha_{p}\left(M, \mathscr{L}_{2}\right) .
\end{aligned}
$$

Thus $\alpha_{p}(M) \equiv \alpha_{p}(M, \mathscr{L})$ is well defined. The same argument shows that $\alpha_{p}$ is a homeomorphism invariant. The case of $\operatorname{dim} M=4$ is similar.

Note 37. If $M$ is smooth, it is possible to give a more classical interpretation of the condition $\alpha_{p}(M)>2$. Namely, if $\alpha_{p}(M)>2$ then, for any $\varepsilon>0$,

$$
\int_{\varepsilon}^{\infty} \int_{\mathscr{F}} \operatorname{tr} e^{-T{\widetilde{\Delta_{p}^{\prime}}}_{p}^{\prime}}(x, x) d \operatorname{vol}(x) d T=\int_{\varepsilon}^{\infty} \operatorname{Tr}_{\Gamma} e^{-T{\widetilde{\Delta_{p}^{\prime}}}_{p}^{\prime}} d T<\infty .
$$

In the case $p=0$, this means that Brownian motion on $\widetilde{M}$ is transient. Similarly, if $\alpha_{p}(M)>4$ then

$$
\begin{aligned}
\int_{\mathscr{F}} & \int_{\widetilde{M}}\left|\int_{\varepsilon}^{\infty} e^{-T \widetilde{\Delta}_{p}^{\prime}}(x, y) d T\right|^{2} d \operatorname{vol}(y) d \operatorname{vol}(x) \\
& =\int_{\varepsilon}^{\infty} \int_{\varepsilon}^{\infty} \int_{\mathscr{F}} \operatorname{tr} e^{-(S+T) \widetilde{\Delta}_{p}^{\prime}}(x, x) d \operatorname{vol}(x) d S d T \\
& =\int_{\varepsilon}^{\infty} \int_{\varepsilon}^{\infty} \operatorname{Tr}_{\Gamma} e^{-(S+T) \widetilde{\Delta}_{p}^{\prime}} d S d T<\infty
\end{aligned}
$$

so $\widetilde{\Delta}_{p}^{\prime-1} e^{-\varepsilon \widetilde{\Delta}_{p}^{\prime}}$ has a kernel given by $\int_{\varepsilon}^{\infty} e^{-T \widetilde{\Delta}_{p}^{\prime}}(x, y) d T$ which is in $L^{2}((\widetilde{M} \times \widetilde{M}) / \Gamma)$.

\section{Abelian fundamental group}

Let $V$ be the vector bundle over $M$ whose fiber over $m \in M$ is $l^{2}\left(\pi^{-1}(m)\right) \cong l^{2}(\Gamma) . \quad V$ is the vector bundle associated to the principal bundle $\Gamma \rightarrow \widetilde{M} \rightarrow M$ by the left regular representation $\rho: \Gamma \rightarrow B\left(l^{2}(\Gamma)\right)$. We can identify $L^{2}(\widetilde{M})$ with $L^{2}$ sections of $V$, and one can think of $\mathscr{T}_{\Gamma}$ as an invariant for the flat Hilbert bundle $V$. If $\pi_{1}(M)$ is abelian, then the left regular representation is a direct integral of one-dimensional 
representations, and $V$ decomposes as an integral of flat unitary line bundles over $M$. This decomposition will allow us to make more precise statements about the Novikov-Shubin invariants.

Let $M$ be a smooth closed connected oriented manifold with $\pi_{1}(M)$ the extension of an abelian group by a finite group. Because $\operatorname{Tr}_{\Gamma} e^{-T \widetilde{\Delta}^{\prime}}$ is multiplicative under finite coverings, $\alpha_{p}(M)$ is invariant under finite coverings, and we may as well assume $\pi_{1}(M)=\mathrm{Z}^{l}$ for some $l$. For a unitary representation $\rho_{\theta}: \mathbf{Z}^{l} \rightarrow \mathrm{U}(1)$ given by $\rho_{\theta}(\vec{n})=e^{i \vec{\theta} \cdot \vec{n}}$, let $E_{\theta}$ be the associated flat line bundle on $M$, with Laplacian $\Delta_{\theta}$. Let $T^{l}$ denote the $l$-torus.

Proposition 38. $\operatorname{Tr}_{\Gamma} e^{-T \widetilde{\Delta}_{p}}=\int_{T^{l}} \operatorname{Tr} e^{-T \Delta_{p, \theta}} d^{l} \theta /(2 \pi)^{l}$.

Proof. Let $a(\vec{n})$ denote the action of $\vec{n} \in \pi_{1}(M)$ on $\widetilde{M}$. Then

$$
\begin{aligned}
\operatorname{Tr}_{\Gamma} e^{-T \widetilde{\Delta}} & =\int_{\mathscr{F}} \operatorname{Tr} e^{-T \widetilde{\Delta}}(x, x) d \operatorname{vol}(x) \\
& =\int_{\mathscr{F}} \sum_{\vec{n}} \int_{T^{l}} \rho_{\theta}(\vec{n}) \operatorname{tr}\left(e^{-T \widetilde{\Delta}} a(\vec{n})^{*}\right)(x, x) d^{l} \theta /(2 \pi)^{l} d \operatorname{vol}(x) \\
& =\int_{T^{l}} \int_{\mathscr{F}} \sum_{\vec{n}} \rho_{\theta}(\vec{n}) \operatorname{tr}\left(e^{-T \widetilde{\Delta}} a(\vec{n})^{*}\right)(x, x) d \operatorname{vol}(x) d^{l} \theta /(2 \pi)^{l} .
\end{aligned}
$$

The change of summations and integrations can be justified by the estimates of the proof of Lemma 4.

We claim

$$
\operatorname{Tr} e^{-T \Delta_{\theta}}=\int_{\mathscr{F}} \sum_{\vec{n}} \rho_{\theta}(\vec{n}) \operatorname{tr}\left(e^{-T \widetilde{\Delta}} a(\vec{n})^{*}\right)(x, x) d \operatorname{vol}(x) .
$$

Then the proposition will follow. To see (81), we can identify the twisted $L^{2}$ forms $\Lambda^{*}\left(M, E_{\theta}\right)$ with $\left\{\omega: \omega\right.$ is a locally $L^{2}$ differential form on $\widetilde{M}$ and $\rho_{\theta}(\vec{n}) a(\vec{n})^{*} \omega=\omega$ for all $\left.\vec{n} \in \pi_{1}(M)\right\}$. The inner product becomes $\langle\omega, \eta\rangle=\int_{\mathscr{F}}\langle\omega(x), \eta(x)\rangle d \operatorname{vol}(x) . \Delta_{\theta}$ is represented by $\omega \rightarrow \widetilde{\Delta} \omega$. Put

$$
K(T, x, y)=\sum_{\vec{n}} \rho_{\theta}(\vec{n})\left(e^{-T \tilde{\Delta}} a(\vec{n})^{*}\right)(x, y) .
$$

Then $K(T, x, y)$ defines an integral operator $K(T)$ on $\Lambda^{*}\left(M, E_{\theta}\right)$ which satisfies $\left(d / d T+\Delta_{\theta}\right) K(T)=0, \lim _{T \rightarrow 0} K(T)=I$. By uniqueness of the heat kernel, it follows that $K(T)=e^{-T \Delta_{\theta}}$, and so

$$
\begin{aligned}
\operatorname{Tr} e^{-T \Delta_{\theta}} & =\int_{M} \operatorname{tr}\left(e^{-T \Delta_{\theta}}\right)(x, x) d \operatorname{vol}(x) \\
& =\int_{\mathscr{F}} \operatorname{tr}(K(T, x, x)) d \operatorname{vol}(x) \text {. q.e.d. }
\end{aligned}
$$


It will be convenient to write all of the operators $\Delta_{\theta}$ as acting on the same Hilbert space. This can be done by choosing a basis $\left\{\tau^{i}\right\}_{i=1}^{l}$ of harmonic 1-forms on $M$ such that if $\gamma$ is a loop representing a class $\vec{n} \in$ $\pi_{1}(M, *)$, then $\int_{\gamma} \vec{\tau}=\vec{n}$. Let $d_{\theta}$ denote the operator $d+i \sum_{j=1}^{l} \theta_{j} e\left(\tau^{j}\right)$. Then $\Delta_{\theta}$ is unitarily equivalent to the selfadjoint operator $d_{\theta}^{*} d_{\theta}+d_{\theta} d_{\theta}^{*}$, (which we will also denote by $\Delta_{\theta}$ ), acting on the Sobolev space $H^{2}(M)$.

Because $\Delta_{\theta}$ is quadratic in $\theta$, it is easy to see that $\left\{\Delta_{\theta}\right\}_{\theta \in T^{l}}$ is an analytic family of type (A) in the sense of [43]. By Theorem XII.13 of [43], the eigenvalues $\lambda_{i}(\theta)$ of $\Delta_{\theta}$ form a sequence of analytic functions locally in $\theta$; they do not necessarily form a sequence of analytic functions on $T^{l}$, but do form a sequence of multivalued functions on $T^{l}$. It follows that for any $\varepsilon>0,\left\{\left(\theta, \lambda_{i}(\theta)\right): \lambda_{i}(\theta) \leq \varepsilon\right\}$ can be written as a union of graphs of a finite number of locally defined analytic functions on $T^{l}$. By taking a sufficiently fine finite covering $\left\{U_{\alpha}\right\}_{\alpha=1}^{s}$ of $\left\{\theta: \lambda_{i}(\theta) \leq \varepsilon\right.$ for some $i\}$, and subordinate partition of unity $\left\{\rho_{\alpha}\right\}_{\alpha=1}^{s}$, we can write

$$
\operatorname{Tr}_{\Gamma} e^{-T \widetilde{\Delta}_{p}}=\sum_{\alpha=1}^{s} \sum_{\beta=1}^{n_{\alpha}} \int_{U_{\alpha}} \rho_{\alpha}(\theta) e^{-T f_{\alpha, \beta}(\theta)} d^{l} \theta /(2 \pi)^{l}+O\left(e^{-\varepsilon T}\right)
$$

for some nonnegative analytic functions $\left\{f_{\alpha, \beta}\right\}_{\beta=1}^{n_{\alpha}}$ defined on $U_{\alpha}$. If $f_{\alpha, \beta}$ is zero in an open subset of $U_{\alpha}$, then it must correspond to an eigenvalue $\lambda_{i}(\theta)$ which vanishes identically on $T^{l}$. This gives a contribution to $\operatorname{Tr}_{\Gamma} e^{-T \widetilde{\Delta}_{p}}$ which is $T$-independent, and so is part of $b_{p}^{(2)}$. Thus

$$
\operatorname{Tr}_{\Gamma} e^{-T \widetilde{\Delta}_{p}}=b_{p}^{(2)}+\sum_{\alpha=1}^{s} \sum_{\beta=1}^{m_{\alpha}} \int_{U_{\alpha}} \rho_{\alpha}(\theta) e^{-T g_{\alpha, \beta}(\theta} d^{l} \theta /(2 \pi)^{l}+O\left(e^{-\varepsilon T}\right),
$$

where the analytic functions $\left\{g_{\alpha, \beta}\right\}_{\beta=1}^{m_{\alpha}}$ are nonnegative and not identically vanishing. If the functions $\left\{g_{\alpha, \beta}\right\}_{\beta=1}^{m_{\alpha}}$ are all bounded away from zero, then $\alpha_{p}(M)=\infty$. Otherwise, by the theory of asymptotic integrals [33],

$$
\operatorname{Tr}_{\Gamma} e^{-T \widetilde{\Delta}_{p}}=b_{p}^{(2)}+O\left(T^{-\alpha_{p} / 2}(\log T)^{k}\right)
$$

for some rational number $\alpha_{p}>0$ and some integer $k$. (Malgrange [33] studies the case of imaginary $T$, but the asymptotics are the same in our case.) Hence we have shown nal.

Proposition 39. If $\pi_{1}(M)$ is abelian, then $\alpha_{p}(M)$ is positive and ratio- 
If $\pi_{1}(M)=\mathbf{Z}$ we can say more. If $\lambda(\theta)$ is a nonnegative nonvanishing analytic function on $U \subset S^{1}$ with an isolated zero at $\theta_{0} \in U$ and $\rho\left(\theta_{0}\right) \neq$ 0 , then $\int_{U} e^{-T \lambda(\theta)} \rho(\theta) d \theta$ is $O\left(T^{-1 /(2 n)}\right)$, where $n$ is the smallest integer such that $\lambda^{(2 n)}\left(\theta_{0}\right) \neq 0$. Thus we have

Proposition 40. If $\pi_{1}(M)=\mathbf{Z}$, then $\alpha_{p}(M)=1 / n_{p}$ for some nonnegative integers $n_{p}$.

We can give conditions for $\lambda^{(2)}\left(\theta_{0}\right)$ to be nonzero using perturbation theory.

Proposition 41. Suppose that for $\theta \in U, \psi(\theta)$ is an analytic family of eigenvectors of $\Delta_{p, \theta}$ with eigenvalue $\lambda(\theta)$, and $\lambda\left(\theta_{0}\right)=0$. Let $\left[\psi\left(\theta_{0}\right)\right]$ denote the class of $\psi\left(\theta_{0}\right)$ in $H^{p}\left(M, E_{\theta_{0}}, \mathbf{R}\right)$, and let $[\tau]$ denote the class of $\tau$ in $H^{1}(M, \mathbf{R})$. Then $\lambda^{(2)}\left(\theta_{0}\right)=0$ iff $[\tau] \cup\left[\psi\left(\theta_{0}\right)\right]=0$ in $H^{p+1}\left(M, E_{\theta_{0}}, \mathbf{R}\right)$ and $[\tau] \cup\left[* \psi\left(\theta_{0}\right)\right]=0$ in $H^{n-p+1}\left(M, E_{\theta_{0}}, \mathbf{R}\right)$.

Proof. We have $d \tau=d^{*} \tau=d_{\theta_{0}} \psi\left(\theta_{0}\right)=d_{\theta_{0}}^{*} \psi\left(\theta_{0}\right)=0$. Then

$$
\begin{aligned}
\left.\frac{d^{2}}{d \theta^{2}}\right|_{\theta=\theta_{0}} \lambda\left(\theta_{0}\right) & =\left.\frac{d^{2}}{d \theta^{2}}\right|_{\theta=\theta_{0}}\left[\left\langle d_{\theta} \psi(\theta), d_{\theta} \psi(\theta)\right\rangle+\left\langle d_{\theta}^{*} \psi(\theta), d_{\theta}^{*} \psi(\theta)\right\rangle\right] \\
& =2\left[\left.\left|\frac{d}{d \theta}\right|_{\theta=\theta_{0}} d_{\theta} \psi(\theta)\right|^{2}+\left.\left|\frac{d}{d \theta}\right|_{\theta=\theta_{0}} d_{\theta}^{*} \psi(\theta)\right|^{2}\right] \\
& =2\left[\left|d_{\theta_{0}} \dot{\psi}+i e(\tau) \psi\right|^{2}+\left|d_{\theta_{0}}^{*} \dot{\psi}-i i(\tau) \psi\right|^{2}\right] .
\end{aligned}
$$

By first-order perturbation theory [43], we have

$$
\dot{\psi}=-G\left(\left.\frac{d}{d \theta}\right|_{\theta=\theta_{0}} \Delta_{\theta}\right) \psi=i G\left(d_{\theta_{0}} i(\tau)-d_{\theta_{0}}^{*} e(\tau)\right) \psi,
$$

where $G$ is the Green's operator for $\Delta_{\theta_{0}}-\lambda\left(\theta_{0}\right)$. Hereafter we will drop the $\theta_{0}$ subscripts. Now

$$
\begin{aligned}
d \dot{\psi}+i e(\tau) \psi=0 & \Leftrightarrow e(\tau) \psi=G d d^{*} e(\tau) \psi \\
& \Leftrightarrow e(\tau) \psi=G\left(d d^{*}+d^{*} d\right) e(\tau) \psi \\
& \Leftrightarrow e(\tau) \psi \perp \operatorname{Ker} \Delta_{p+1, \theta_{0}} .
\end{aligned}
$$

Because $d e(\tau) \psi=0, e(\tau) \psi$ can be written as $\eta+d \rho$ for some $\eta \in$ $\operatorname{Ker} \Delta_{p+1, \theta_{0}}, \rho \in \Lambda^{p}\left(M, E_{\theta_{0}}\right)$. Then 


$$
\begin{aligned}
e(\tau) \psi \perp \operatorname{Ker} \Delta_{p+1, \theta_{0}} & \Leftrightarrow \eta=0 \Leftrightarrow e(\tau) \psi \in \operatorname{Im} d \\
& \Leftrightarrow[e(\tau) \psi]=0 \quad \text { in } H^{p+1}\left(M, E_{\theta_{0}}, \mathbf{R}\right) \\
& \Leftrightarrow[\tau] \cup[\psi]=0 \quad \text { in } H^{p+1}\left(M, E_{\theta_{0}}, \mathbf{R}\right) .
\end{aligned}
$$

The same arguments, applied to $* \psi$ instead of $\psi$, give

$$
d * \dot{\psi}-i(\tau) \psi=0 \Leftrightarrow[\tau] \cup[* \psi]=0 \text { in } H^{n-p+1}\left(M, E_{\theta_{0}}, \mathbf{R}\right) \text {. q.e.d. }
$$

Using higher order perturbation theory, one can show that given $\lambda^{(2)}\left(\theta_{0}\right)$ $=0$, one has $\lambda^{(4)}\left(\theta_{0}\right)=0$ iff the Massey products $\langle[\tau],[\psi],[\tau]\rangle$ and $\langle[\tau],[* \psi],[\tau]\rangle$ vanish, that is, there is a $p$-form $\psi^{(1)}$ such that $\tau \wedge \psi=$ $d \psi^{(1)}$ and $\left[\tau \wedge \psi^{(1)}\right]=0(\bmod [\tau])$, and similarly for $* \psi$. By redefining $\psi^{(1)}$, the vanishing of $\langle[\tau],[\psi],[\tau]\rangle$ is equivalent to the existence of $p$ forms $\psi^{(1)}$ and $\psi^{(2)}$ such that $\tau \wedge \psi=d \psi^{(1)}$ and $\tau \wedge \psi^{(1)}=d \psi^{(2)}$. One can see the pattern that, in general, $\lambda^{(2)}\left(\theta_{0}\right)=\lambda^{(4)}\left(\theta_{0}\right)=\cdots=\lambda^{(2 r)}\left(\theta_{0}\right)=0$ iff

$$
\begin{aligned}
& \exists p \text {-forms } \psi^{(1)}, \psi^{(2)}, \cdots, \psi^{(r)} \text { such that } \\
& \tau \wedge \psi=d \psi^{(1)}, \tau \wedge \psi^{(1)}=d \psi^{(2)}, \cdots, \tau \wedge \psi^{(r-1)}=d \psi^{(r)},
\end{aligned}
$$

and similarly for $* \psi$. The condition $(*)$ can be re-expressed in a way independent of choices as

$$
\begin{aligned}
& \exists p \text {-forms } \psi^{(1)}, \psi^{(2)}, \cdots, \psi^{(r)} \text { such that } \tau \wedge \psi=d \psi^{(1)}, \\
& \tau \wedge \psi^{(1)}=d \psi^{(2)}(\bmod \tau), \cdots, \tau \wedge \psi^{(r-1)}=d \psi^{(r)}(\bmod \tau) .
\end{aligned}
$$

Example 42. Let $M$ be the total space of a nontrivial $\mathrm{SU}(2)$ bundle over $B=S^{1} \times S^{3}$. We will show that $\alpha_{3}(M)=1 / 2$. By the Gysin sequence for twisted cohomology,

$$
\begin{aligned}
\cdots \rightarrow H^{k}\left(B, E_{\theta}, \mathbf{R}\right) & \rightarrow H^{k}\left(M, \pi^{*} E_{\theta}, \mathbf{R}\right) \rightarrow H^{k+1-3}\left(B, E_{\theta}, \mathbf{R}\right) \\
& \rightarrow H^{k+1}\left(B, E_{\theta}, \mathbf{R}\right) \rightarrow \cdots
\end{aligned}
$$

$H^{3}\left(M, \pi^{*} E_{\theta}, \mathbf{R}\right)$ is nonzero only for $\theta=0$ : By the Hirsch lemma, the minimal model of $M$ has a generator $x$ in degree 1 and generators $y_{1}$ and $y_{2}$ in degree 3, with the relations $d x=0, d y_{1}=0$, and $d y_{2}=x y_{1}$ [22]. Taking $[\tau]=[x]$ and $[\psi]=\left[y_{1}\right]$, we have $[* \psi]=\left[x y_{2}\right]$. Then $[\tau] \cup[\psi]=$ 0 in $H^{4}(M, \mathbf{R})$ and $[\tau] \cup[* \psi]=0$ in $H^{5}(M, \mathbf{R})$, so $\lambda^{(2)}\left(\theta_{0}\right)=0$. On the other hand, taking $\psi^{(1)}=y_{2}$, we have $\left[\tau \wedge \psi^{(1)}\right] \neq 0$ in $H^{4}(M, \mathbf{R})$, 
so $\lambda^{(4)}\left(\theta_{0}\right) \neq 0$. Thus $\alpha_{3}(M)=1 / 2$. This example shows that $\alpha_{p}(M)$ can be noninteger. Also, by comparing with $\alpha_{3}\left(S^{1} \times S^{3} \times S^{3}\right)=1$ and $\alpha_{3}\left(S^{1} \times S^{6}\right)=\infty$, it is clear that for general $p, \alpha_{p}(M)$ depends on more than just the dimension and fundamental group of $M$. Repeatedly forming $\mathrm{SU}(2)$ principal bundles, we can construct a manifold $M^{3 r+1}$ whose minimal model has a generator $x$ in degree 1 and generators $y_{1}, \cdots, y_{r}$ in degree 3, with the relations $d x=0, d y_{1}=0, d y_{2}=x y_{1}, \cdots, d y_{r}=$ $x y_{r-1}$. Then $\alpha_{3}\left(M^{3 r+1}\right)=1 / r$. This shows that $\alpha_{p}(M)$ can be arbitrarily close to 0 .

If $\pi_{1}(M)=\mathbf{Z}^{l}$ and $H^{p}\left(M, E_{\theta_{0}}, \mathbf{R}\right) \neq 0$, one can use perturbation theory to determine the asymptotics of $\lambda(\theta)$ around $\theta=\theta_{0}$. However, since the procedure to obtain the asymptotics of $\int_{U} e^{-T \lambda(\theta)} \rho(\theta) d \theta$ is nonconstructive and involves blowing up singularities, it does not seem to be much use in determining $\alpha_{p}(M)$ explicitly. In the special case when $\lambda(\theta)$ is asymptotically a positive definite quadratic function around $\theta_{0}$, $\int_{U} e^{-T \lambda(\theta)} \rho(\theta) d \theta$ is asymptotically $O\left(T^{-l / 2}\right)$. By the same arguments as for Proposition 41, this will be the case iff for all $[\tau] \in H^{1}(M, \mathbf{R})$, $[\tau] \cup[\psi]=[\tau] \cup[* \psi]=0$ implies $[\tau]=0$.

Proposition 43. If $\pi_{1}(M)=\mathbf{Z}^{l}$, then $\alpha_{0}=\alpha_{1}=l$.

Proof. The only value of $\theta$ where $H^{0}\left(M, E_{\theta}, \mathbf{R}\right)$ or $H^{1}\left(M, E_{\theta}, \mathbf{R}\right)$ is nonzero is $\theta=0$. For $p=0$, we have $[\psi]=[1] \in H^{0}(M, \mathbf{R})$, and so for $[\tau] \in H^{1}(M, \mathbf{R}),[\tau] \cup[\psi]=0$ implies $[\tau]=0$. For $p=1$, take a nonzero $[\psi] \in H^{1}(M, \mathbf{R})$ and suppose that for some $[\tau] \in H^{1}(M, \mathbf{R})$, $[\tau] \cup[\psi]=[\tau] \cup[* \psi]=0$. From $[\tau] \cup[\psi]=0,[\tau]$ must be proportionate to $[\psi]$, but then by Poincaré duality $[\tau] \cup\left[{ }^{*} \psi\right]=0$ implies $[\tau]=0$.

Note 44. That $\alpha_{0}$ is $l$ is part of a more general result (see the next section).

Note 45. The arguments in this section also work for an arbitrary infinite normal Abelian cover of a closed oriented manifold $M$. When applied to the $L^{2}$ analytic torsion of a manifold with infinite Abelian fundamental group, they give

$$
\mathscr{T}_{\Gamma}(M)=\int_{T^{l}} T(\theta) d^{l} \theta /(2 \pi)^{l},
$$

where $T(\theta)$ is the ordinary analytic torsion of the flat line bundle specified by $\theta$. In particular, the results of [38] and the identity

$$
\int_{S^{1}} \ln \left|a-e^{i \theta}\right| d \theta / 2 \pi=\ln |a| \theta(|a|-1)
$$


give that if $f$ is a diffeomorphism of $N, N$ simply-connected, and $M$ is the mapping cylinder, then up to a constant,

$$
\mathscr{T}_{\Gamma}(M)=\sum \ln \left(\left|\lambda_{i, \text { even }}\right|\right)-\sum \ln \left(\left|\lambda_{i, \text { odd }}\right|\right),
$$

where $\left\{\lambda_{\text {ieven }}\right\}$ are the eigenvalues of the action of $H^{*}(f)$ on $H^{\text {even }}(N)$ of norm greater than one, and similarly for $\left\{\lambda_{i, \text { odd }}\right\}$.

\section{Examples of $\alpha_{p}$}

A. $\mathbf{p}=\mathbf{0}$. Varopoulos [49] has shown $\alpha_{0}=\infty$ unless $\pi_{1}(M)$ has polynomial growth (i.e., $\pi_{1}(M)$ is almost nilpotent [24]), in which case $\alpha_{0}$ is the growth rate of $\pi_{1}(M)$.

B. Locally symmetric spaces. We collect some properties of the spectrum of the Laplacian on differential forms on irreducible noncompact Riemannian symmetric spaces $\widetilde{M}=G / K$.

$\widetilde{M}$ can have harmonic $p$-forms only for $p=\operatorname{dim}(\widetilde{M}) / 2$ [3, II, 5.4]. These correspond to discrete series representations of $G$, and so can only occur for $\operatorname{rank} G=\operatorname{rank} K$. Furthermore, if $\operatorname{rank} G=\operatorname{rank} K$, then $\chi(M) \neq 0$ [5], and so by the $L^{2}$ index theorem [1], such harmonic $p$ forms do occur.

To convert to the notation of [3], the Laplacian $\widetilde{\Delta}_{p}$ acting on the smooth vectors in $\Lambda^{p}(\widetilde{M})$ is equivalent to $\Delta$ acting on $C^{p}(g, k, H)$, where $H$ is the unitary $(g, k)$-module of smooth vectors in $L^{2}(G)$. By Kuga's Lemma, the action of $\Delta$ on $C^{p}(g, k, H)$ is given by the representation of the Casimir $C$ of $g$ on $H$.

In order to know when $\alpha_{p}(M)$ can possibly be finite, one can ignore the discrete spectrum of $\widetilde{\Delta}_{p}$, as its contribution to $\operatorname{tr} e^{-T \widetilde{\Delta}_{p}(x, x)}$ will either be constant in $T$ or decrease exponentially in $T$. Then a necessary condition for $\alpha_{p}(M)$ to be finite is that the continuous spectrum of $\widetilde{\Delta}_{p}$ extend down to 0 . The continuous spectrum corresponds to the part of the regular representation of $G$ induced from cuspidal parabolic subgroups, and so the question is whether an induced representation $V$ can give a vanishing representation of the Casimir operator. We can assume that the induced representation is irreducible, upon which having vanishing Casimir is equivalent to $C^{p}(g, h, V)$ consisting of harmonic cochains, which is equivalent to $H^{p}(g, k, V)$ being nonzero [3, II, 3.1]. By [3, III, 5.1], for the tempered representations $V$ which are of interest to us, $H^{p}(g, k, V)=0$ if $p \notin$ $[\operatorname{dim}(\widetilde{M}) / 2-(\operatorname{rank}(G)-\operatorname{rank}(K)) / 2, \operatorname{dim}(\widetilde{M}) / 2+(\operatorname{rank}(G)-\operatorname{rank}(K)) / 2]$ 
Thus $\alpha_{p}(M)=\infty$ for such $p$. Furthermore, $H^{p}(g, k, V)=0$ if $V$ is not a fundamental series representation. Thus if $\operatorname{rank}(G)=\operatorname{rank}(K)$, then $\alpha_{p}(M)=\infty$ for all $p$.

Precise information on the representations $V$ with nonvanishing $(g, k)$-cohomology is given in [51], to which we refer for details. In particular, it follows from [51] that the continuous spectrum of $\widetilde{\Delta}_{p}$ does extend down to 0 if $p \in[\operatorname{dim}(\widetilde{M}) / 2-(\operatorname{rank}(G)-\operatorname{rank}(K)) / 2, \operatorname{dim}(\widetilde{M}) / 2+$ $(\operatorname{rank}(G)-\operatorname{rank}(K)) / 2$ ] , due to the existence of a unique fundamental series representation $V$. Then $\alpha_{p}(M)$ will be finite and independent of $p$ in this range, and can be computed using the Plancherel formula. We will only give the result for the simplest case.

Proposition 46. If $M$ is a real hyperbolic manifold of odd dimension $d$, then $\alpha_{p}(M)=\infty$ if $p \neq(d \pm 1) / 2$ and $\alpha_{p}(M)=1$ if $p=(d \pm 1) / 2$.

Proof. By the above discussion, or Proposition 15, if $j \neq(d-1) / 2$, then the spectrum of $\widetilde{\Delta}$ on $L^{2}$ coclosed $j$-forms is bounded away from zero, and $F_{j}(T)$ has exponential decay. For $j=(d-1) / 2$,

$$
\begin{aligned}
F_{j}(T) & =(4 \pi)^{-d / 2} \frac{1}{\Gamma(d / 2)}\left(\frac{d-1}{2}\right) \int_{-\infty}^{\infty} e^{-T \nu^{2}} \prod_{i=1}^{(d-1) / 2}\left(\nu^{2}+i^{2}\right) d \nu \\
& =O\left(\int_{-\infty}^{\infty} e^{-T \nu^{2}} \prod_{i=1}^{(d-1) / 2} i^{2} d \nu\right)=O\left(T^{-1 / 2}\right) .
\end{aligned}
$$

Hence the proposition follows. q.e.d.

A generalization of the above results, when applied to Hermitian or quaternionic noncompact symmetric spaces, is given by the following propositions.

Definition 47 [26]. A closed Kähler manifold $M$ is Kähler hyperbolic if the Kähler form $\omega$ on $\widetilde{M}$ can be written as $\omega=d \eta$ for some bounded 1-form $\eta$ on $\widetilde{M}$.

Proposition 48 [26]. If $M$ is a Kähler hyperbolic manifold, then the spectrum of $\widetilde{\Delta}$ on $(\operatorname{Ker} \widetilde{\Delta})^{\perp}$ is bounded away from zero.

Definition 49. Suppose that $M$ is a closed quaternionic-Kähler manifold. $M$ is quaternionic-Kähler hyperbolic if the canonical 4-form $\omega$ on $\widetilde{M}$ can be written as $\omega=d \eta$ for some bounded 3 -form $\eta$ on $\widetilde{M}$.

Proposition 50. If $M$ is quaternionic-Kähler hyperbolic, then the spectrum of $\widetilde{\Delta}_{p}$ on $\left(\operatorname{Ker} \widetilde{\Delta}_{p}\right)^{\perp}$ is bounded away from zero for $p$ even and $p \neq(\operatorname{dim} M) / 2$.

Proof. Using the Lefschetz isomorphism on a quaternionic-Kähler manifold [2], the proof is the same as that of [26]. 
C. Heisenberg groups. Suppose that one has a Lie group $G$ with a left-invariant orthonormal basis of the tangent space. Then there is a left-invariant orthonormal basis of $\Lambda^{*}(G)$, and in terms of this basis the Laplacian becomes a matrix of differential operators on $G$. One can hope to compute heat kernels using harmonic analysis on $G$. In particular, on the Heisenberg groups the analysis simplifies because of the CR structure. The author wishes to thank Mike Christ for much help with the results of this section.

Let $M^{2 m+1}$ be a closed oriented manifold which is homeomorphic to a quotient $G / \Gamma$ of the Heisenberg group by a discrete subgroup. The Heisenberg group $G$ can be identified with $\mathbf{R}^{2 m+1}$, with coordinates $\left\{\left\{x^{j}\right\}_{j=1}^{m}\right.$, $\left.\left\{y^{j}\right\}_{j=1}^{m}, w\right\}$. A basis of left-invariant vector fields is given by

$$
X_{j}=\partial_{x^{j}}-\frac{1}{2} y^{j} \partial_{w}, \quad Y_{j}=\partial_{y^{j}}+\frac{1}{2} x^{j} \partial_{w}, \quad W=\frac{1}{c} \partial_{w}
$$

for any $c \in \mathbf{R}-\{0\}$. We will consider the left-invariant metric on $G$ given by requiring that $\left\{X_{j}, Y_{j}, W\right\}$ be an orthonormal basis. It is convenient to complexify and put

$$
Z_{j}=\frac{1}{\sqrt{2}}\left(X_{j}-i Y_{j}\right), \quad Z_{\bar{j}}=\frac{1}{\sqrt{2}}\left(X_{j}+i Y_{j}\right) .
$$

Then a Hermitian basis for $T^{*} G \otimes C$ is given by

$$
\begin{aligned}
\tau^{j} & =\frac{1}{\sqrt{2}}\left(d x^{j}+i d y^{j}\right), \quad \tau^{\bar{j}}=\frac{1}{\sqrt{2}}\left(d x^{j}-i d y^{j}\right), \\
\tau^{w} & =c\left(d w-\frac{1}{2} \sum_{j} x^{j} d y^{j}+\frac{1}{2} \sum_{j} y^{j} d x^{j}\right) .
\end{aligned}
$$

The differentials of the basis elements are

$$
d \tau^{j}=d \tau^{\bar{j}}=0, \quad d \tau^{w}=-i c \sum_{j} \tau^{j} \wedge \tau^{\bar{j}} .
$$

Using the global orthonormal basis of $\Lambda^{*} G \otimes \mathbf{C}$, we write differential operators on $\Lambda^{*} G \otimes \mathbf{C}$ as matrices of differential operators on $C^{\infty}(G) \otimes \mathbf{C}$. In particular, $e\left(\tau^{j}\right), e\left(\tau^{\bar{j}}\right), e\left(\tau^{w}\right), i\left(Z_{j}\right), i\left(Z_{\bar{j}}\right)$, and $i(W)$ are represented as matrices of zeros and ones. One can compute that the covariant 
derivatives $\nabla: T G \otimes \Lambda^{*}(G) \otimes \mathbf{C} \rightarrow \Lambda^{*}(G) \otimes \mathbf{C}$ are given by

$$
\begin{aligned}
& \nabla_{Z_{j}}=Z_{j}-\frac{1}{2} i c e\left(\tau^{\bar{j}}\right) i(W)+\frac{1}{2} i c e\left(\tau^{w}\right) i\left(Z_{j}\right), \\
& \nabla_{Z_{j}}=Z_{\bar{j}}+\frac{1}{2} i c e\left(\tau^{j}\right) i(W)-\frac{1}{2} i c e\left(\tau^{w}\right) i\left(Z_{\bar{j}}\right), \\
& \nabla_{W}=W+\frac{1}{2} i c \sum_{k}\left(e\left(\tau^{k}\right) i\left(Z_{k}\right)-e\left(\tau^{\bar{k}}\right) i\left(Z_{\bar{k}}\right)\right) .
\end{aligned}
$$

Proposition 51. Let $\mathscr{S}^{p}$ denote the G-invariant subspace of $\Lambda^{p}(T G) \otimes$ $\mathbf{C}$ defined by

$$
\begin{aligned}
\mathscr{S}^{p}=\left\{\omega \in \Lambda^{p}(T G) \otimes \mathbf{C}: 0\right. & =i(W) \omega=i\left(Z_{\bar{j}}\right) \omega \\
& \left.=Z_{\bar{j}} \omega \text { for all } j=1, \cdots, m\right\} .
\end{aligned}
$$

Then $\mathscr{S}^{p}$ is an invariant subspace for the Laplacian, and $\Delta=-W^{2}+$ ic $(m-p) W$ on $\mathscr{S}^{p}$.

Proof. We have

$$
d \omega=\sum_{j}\left[e\left(\tau^{j}\right) \nabla_{Z_{j}}+e\left(\tau^{\bar{j}}\right) \nabla_{Z_{\bar{j}}}+e\left(\tau^{w}\right) \nabla_{W}\right]
$$

and

$$
d^{*} \omega=-\sum_{j}\left[i\left(Z_{\bar{j}}\right) \nabla_{Z_{j}}+i\left(Z_{j}\right) \nabla_{Z_{\bar{j}}}+i(W) \nabla_{W}\right] \omega
$$

on $\Lambda^{*}(G) \otimes C$, and

$$
\begin{aligned}
& i(W) e\left(\tau^{w}\right) W \omega=W \omega, \quad \sum_{k} e\left(\tau^{k}\right) i\left(Z_{k}\right) \omega=p \omega, \\
& \nabla_{Z_{j}} \omega=Z_{j} \omega+\frac{1}{2} i c e\left(\tau^{w}\right) i\left(Z_{j}\right) \omega \\
& \nabla_{Z_{\bar{j}}} \omega=0 \text { and } \nabla_{W} \omega=W \omega+\frac{1}{2} i c p \omega
\end{aligned}
$$

on $\mathscr{S}^{p}$. Then on $\mathscr{S}^{p}$,

$$
\begin{aligned}
d \omega & =\sum_{j}\left[e\left(\tau^{j}\right) Z_{j} \omega-\frac{1}{2} i c e\left(\tau^{w}\right) e\left(\tau^{j}\right) i\left(Z_{j}\right) \omega\right]+e\left(\tau^{w}\right) W \omega+\frac{1}{2} i c p e\left(\tau^{w}\right) \omega \\
& =\sum_{j} e\left(\tau^{j}\right) Z_{j} \omega+e\left(\tau^{w}\right) W \omega
\end{aligned}
$$

and

$$
d^{*} \omega=-\sum_{j} i\left(Z_{\bar{j}}\right) Z_{j} \omega-i(W) W \omega-\frac{1}{2} i c p i(W) \omega=0
$$


So for $\omega \in \mathscr{S}^{p}$,

$$
\begin{aligned}
\Delta \omega=d^{*} d \omega= & -\sum_{j, k} i\left(Z_{k}\right) e\left(\tau^{j}\right) Z_{\bar{k}} Z_{j} \omega \\
& +\sum_{k}\left[\frac{1}{2} i c i\left(Z_{\bar{k}}\right) e\left(\tau^{\bar{k}}\right) i(W) e\left(\tau^{w}\right) W \omega\right. \\
& \quad-\frac{1}{2} i c i\left(Z_{k}\right) e\left(\tau^{k}\right) i(W) e\left(\tau^{w}\right) W \omega \\
& \left.-\frac{1}{2} i c i(W) e\left(\tau^{k}\right) i\left(Z_{k}\right) e\left(\tau^{w}\right) W \omega\right] \\
& -i(W) e\left(\tau^{w}\right) W^{2} \omega .
\end{aligned}
$$

Using (105), we obtain

$$
\begin{aligned}
\Delta \omega & =-W^{2} \omega-\sum_{j, k} i\left(Z_{k}\right) e\left(\tau^{j}\right) Z_{\bar{k}} Z_{j} \omega \\
& =-W^{2} \omega-\sum_{j, k} i\left(Z_{k}\right) e\left(\tau^{j}\right)\left[Z_{\bar{k}}, Z_{j}\right] \omega \\
& =-W^{2} \omega+\sum_{k} i c i\left(Z_{k}\right) e\left(\tau^{k}\right) W \omega=-W^{2} \omega+i c(m-p) W \omega .
\end{aligned}
$$

\section{Proposition 52.}

$$
\alpha_{p}(M) \leq \begin{cases}2(m+1), & p \neq m, m+1, \\ m+1, & p=m, m+1\end{cases}
$$

(By part A, this inequality is exact for $p=0,2 m+1$. )

Proof. We can assume $p \leq m$. Because $\mathscr{S}^{p}$ is invariant under $\widetilde{\Delta}$,

$$
\operatorname{Tr}_{\Gamma} e^{-T \widetilde{\Delta}_{p}^{\prime}} \geq\left.\operatorname{Tr}_{\Gamma} e^{-T \widetilde{\Delta}_{p}^{\prime}}\right|_{\mathscr{S}^{p}}=\operatorname{vol}(M) \operatorname{tr} \exp \left(-\left.T \widetilde{\Delta}_{p}^{\prime}\right|_{\mathscr{S}^{p}}\right)(x, x)
$$

Define

$$
\mathscr{H}=\left\{\omega \in L^{2}(G): Z_{\bar{j}} \omega=0 \text { for } j=1, \cdots, m\right\}
$$

Then $\mathscr{S}^{p} \cong \mathscr{H} \otimes \mathbf{C}^{\left(\begin{array}{c}m \\ p\end{array}\right)}$ and

$$
\begin{aligned}
& \operatorname{tr} \exp \left(-\left.T \widetilde{\Delta}_{p}^{\prime}\right|_{\mathscr{S}^{p}}\right)(x, x) \\
& \quad=\left(\begin{array}{c}
m \\
p
\end{array}\right)\left(\exp \left(-\left.T\left(-W^{2}+i c(m-p) W\right)\right|_{\mathscr{H}}\right)\right)(x, x) .
\end{aligned}
$$

If $P$ denotes the Szegö projection, the orthogonal projection from $L^{2}(G)$ to $\mathscr{H}$, then

$$
\begin{aligned}
{[\exp (} & \left.\left.-\left.T\left(-W^{2}+i c(m-p) W\right)\right|_{\mathscr{E}}\right)\right](x, x) \\
& =\left[\left(\exp \left(-T\left(-W^{2}+i c(m-p) W\right)\right)\right) P\right](x, x) \\
& =\int_{G}\left[\exp \left(-T\left(-W^{2}+i c(m-p) W\right)\right)\right](x, y) P(y, x) d \operatorname{vol}(y)
\end{aligned}
$$


It is convenient to take the Fourier transform in the $w$ direction, giving

$$
\int_{\mathbf{R}} e^{-T\left(k^{2} / c^{2}+(m-p) k\right)} \widehat{P}(\vec{x}, \vec{x}, k) d k /(2 \pi c),
$$

where $\vec{x} \in \mathbf{R}^{2 m}$.

Now $\widehat{P}(\vec{x}, \vec{x}, k)$ is proportionate to $k^{m} \theta(k)$. This can be seen by writing $e^{2 T \sum_{j} Z_{j} Z_{\bar{j}}}$ as $e^{-T\left(\Delta_{K}-i c m W\right)}$ and taking the $T \rightarrow \infty$ limit of the expression for $e^{-T \Delta_{K}}$ in [21], where $\Delta_{K}=-\sum_{j}\left(Z_{j} Z_{\bar{j}}+Z_{\bar{j}} Z_{j}\right)$. Thus we have something proportionate to $\int_{0}^{\infty} k^{m} e^{-T\left(k^{2} / c^{2}+(m-p) k\right)} d k$. If $m-p>$ 0 , the large-T asymptotics is given by $\int_{0}^{\infty} k^{m} e^{-T(m-p) k} d k=O\left(T^{-m-1}\right)$. If $m-p=0$, the large-T asymptotics is given by $\int_{0}^{\infty} k^{m} e^{-T k^{2} / c^{2}} d k=$ $O\left(T^{-(m+1) / 2}\right)$.

Proposition 53. If $m=1$, i.e., $M$ is a three-dimensional Nil manifold, then $\alpha_{0}(M)=4$ and $\alpha_{1}(M)=2$.

Proof. By part A, the assertion for $\alpha_{0}$ is true, and we only have to consider $p=1$. A computation gives that in the orthonormal basis of $\Lambda^{1}(G) \otimes \mathbf{C}$ given by $\left\{\tau^{z}, \tau^{\bar{z}}, \tau^{w}\right\}$, the Laplacian takes the form

$$
\Delta_{1}=\left(\begin{array}{ccc}
-2 Z \bar{Z}-W^{2} & 0 & -i c Z \\
0 & -2 \bar{Z} Z-W^{2} & i c \bar{Z} \\
-i c \bar{Z} & i c Z & -(Z \bar{Z}+\bar{Z} Z)+c^{2}-W^{2}
\end{array}\right),
$$

and the subspace $\mathscr{S}^{p}$ of Proposition 51 is the space of vectors

$$
\left(\begin{array}{l}
\eta \\
0 \\
0
\end{array}\right)
$$

with $\bar{Z} \eta=0$.

In order to compute the spectral decomposition of $\Delta_{1}$, let us first consider the Laplacian

$$
\Delta_{0}=-(Z \bar{Z}+\bar{Z} Z)-W^{2}
$$

acting on $L^{2}(G) \otimes \mathbf{C}$. By taking a Fourier transform in the $w$ direction, we can reduce to the study of the operators

$$
\Delta_{0}(k)=-(Z \bar{Z}+\bar{Z} Z)+(k / c)^{2}
$$

acting on $L^{2}\left(\mathbf{R}^{2}\right) \otimes \mathbf{C}$, where $[Z, \bar{Z}]=k$. For $k>0$, define the operators

$$
a=i k^{-1 / 2} \bar{Z} \text { and } a^{*}=i k^{-1 / 2} Z \text {. }
$$


Then $a^{*}$ and $a$ are adjoint operators and satisfy the CCR (canonical commutation relation) $\left[a, a^{*}\right]=1$, and $\Delta_{0}(k)=k\left(2 a^{*} a+1\right)+(k / c)^{2}$. From the representation theory of the CCR we know that $L^{2}\left(\mathbf{R}^{2}\right) \otimes \mathbf{C}$ is the direct sum of Fock spaces built up on the vacuum $\operatorname{Ker}(a)=\operatorname{Ker}(\bar{Z})$. That is, if $\left\{v_{l, k}\right\}_{l=0}^{\infty}$ is an orthonormal basis for $\operatorname{Ker}(\bar{Z})$, and we define $\psi_{m l k}=$ $\frac{1}{\sqrt{m !}}\left(a^{*}\right)^{m} v_{l, k}$, then $\left\{\psi_{m l k}\right\}_{l, m=0}^{\infty}$ is an orthonormal basis for $L^{2}\left(\mathbf{R}^{2}\right) \otimes \mathbf{C}$, with

$$
\Delta_{0}(k) \psi_{m l k}=\left[(2 m+1) k+(k / c)^{2}\right] \psi_{m l k} .
$$

In turn, $\operatorname{Ker}(\bar{Z})$ is also a Fock space. This can be seen by writing

$$
\bar{Z}=\sqrt{2} e^{-k z \bar{z} / 4} \partial_{\bar{z}} e^{k z \bar{z} / 4},
$$

so that $\operatorname{Ker}(\bar{Z})$ is the space of functions $f(z) e^{-k z \bar{z} / 4}$ in $L^{2}\left(\mathbf{R}^{2}, \frac{i}{2} d z \wedge\right.$ $d \bar{z}) \otimes \mathbf{C}$, or equivalently the Bargmann space $\mathfrak{B}$ of holomorphic functions $f(z)$ in $L^{2}\left(\mathbf{R}^{2}, \frac{i}{2} e^{-k z \bar{z} / 2} d z \wedge d \bar{z}\right) \otimes \mathbf{C}$. Define the operators

$$
b^{*}=(k / 2)^{1 / 2} z \text { and } b=(k / 2)^{-1 / 2} \partial_{z} .
$$

Then $b^{*}$ and $b$ are adjoint operators on $\mathfrak{B}$ and satisfy the CCR. Thus an orthonormal basis for $\mathfrak{B}$ is given by $\left(b^{*}\right)^{l} \sqrt{k / 2 \pi} / \sqrt{l !}$, and an orthonormal basis for $\operatorname{Ker}(\bar{Z})$ is given by

$$
v_{l, k}=\sqrt{k /(2 \pi l !)}(\sqrt{k / 2} z)^{l} e^{-k z \bar{z} / 4}
$$

In conclusion, an orthonormal basis of $L^{2}\left(\mathbf{R}^{2}\right) \otimes \mathbf{C}$ is given by

$$
\psi_{m l k}=\frac{1}{\sqrt{m !}}\left(\frac{i}{\sqrt{k}} \sqrt{2}\left(\partial_{z}-\frac{k \bar{z}}{4}\right)\right)^{m} \sqrt{\frac{k}{(2 \pi l !)}}\left(\sqrt{\frac{k}{2} z}\right)^{l} e^{-k z \bar{z} / 4}
$$

Similarly, if $k<0$, define

$$
a^{*}=i|k|^{-1 / 2} \bar{Z} \text { and } a=i|k|^{-1 / 2} Z \text {. }
$$

Thus $\Delta_{0}(k)=|k|\left(2 a^{*} a+1\right)+(k / c)^{2}$, and an orthonormal basis of $L^{2}\left(\mathbf{R}^{2}\right) \otimes$ $\mathrm{C}$ is given by

$$
\psi_{m l k}=\frac{1}{\sqrt{m !}}\left(\frac{i \sqrt{2}}{\sqrt{|k|}}\left(\partial_{\bar{z}}+\frac{k z}{4}\right)\right)^{m} \sqrt{\frac{|k|}{2 \pi l !}}\left(\sqrt{\frac{|k|}{2}} \bar{z}\right)^{l} e^{-|k| z \bar{z} / 4}
$$

with

$$
\Delta_{0}(k) \psi_{m l k}=\left[(2 m+1)|k|+(k / c)^{2}\right] \psi_{m l k}
$$


As an example, we can compute

$$
e^{-T \Delta_{0}}(0,0)=\int_{-\infty}^{\infty} \frac{d k}{2 \pi c} \sum_{l, m=0}^{\infty} e^{-T\left[|k|(2 m+1)+k^{2} / c^{2}\right]}\left|\psi_{m l k}(0)\right|^{2}
$$

One can see that

$$
\psi_{m l k}(0)=i^{m}(|k| / 2 \pi)^{1 / 2} \delta_{m, l}
$$

and so

$$
e^{-T \Delta_{0}}(0,0)=\int_{0}^{\infty} \frac{d k}{2 \pi c} \frac{k}{2 \pi} e^{-T k^{2} / c^{2}}(\operatorname{Sinh} T k)^{-1}
$$

In particular, changing variables to $u=T k$, we have

$$
\begin{aligned}
e^{-T \Delta_{0}}(0,0) & =\left(4 \pi^{2} c T^{2}\right)^{-1} \int_{0}^{\infty} u d u e^{-T^{-1} u^{2} / c^{2}}(\operatorname{Sinh} u)^{-1} \\
& =O\left(T^{-2}\right) \text { as } T \rightarrow \infty,
\end{aligned}
$$

showing that $\alpha_{0}(M)=4$.

In order to analyze $\Delta_{1}$, let us take the Fourier transform in the $w$ direction and define

$$
\Delta_{1}(k)=\left(\begin{array}{ccc}
-2 Z \bar{Z}+\frac{k^{2}}{c^{2}} & 0 & -i c Z \\
0 & -2 \bar{Z} Z+\frac{k^{2}}{c^{2}} & i c \bar{Z} \\
-i c \bar{Z} & i c Z & -Z \bar{Z}-\bar{Z} Z+c^{2}+\frac{k^{2}}{c^{2}}
\end{array}\right),
$$

acting on $\left(L^{2}\left(\mathbf{R}^{2}\right) \otimes \mathbf{C}^{3}\right)$. Considering first the case $k>0$, we can write $\Delta_{1}(k)$ in terms of creation and annihilation operators as

$$
\Delta_{1}(k)=\left(\begin{array}{ccc}
2 k a^{*} a+\frac{k^{2}}{c^{2}} & 0 & -c k^{1 / 2} a^{*} \\
0 & 2 k a a^{*}+\frac{k^{2}}{c^{2}} & c k^{1 / 2} a \\
-c k^{1 / 2} a & c k^{1 / 2} a^{*} & k\left(a^{*} a+a a^{*}\right)+c^{2}+\frac{k^{2}}{c^{2}}
\end{array}\right) .
$$

Using the basis of $L^{2}\left(\mathbf{R}^{2}\right) \otimes \mathbf{C}$ given by (123), we have a basis of $\left(L^{2}\left(\mathbf{R}^{2}\right) \otimes\right.$ C) $)^{3}$ given by $\left(\psi_{m_{1} l_{1} k}, 0,0\right),\left(0, \psi_{m_{2} l_{2} k}, 0\right)$, and $\left(0,0, \psi_{m_{3} l_{3} k}\right)$. From the structure of $\Delta_{1}(k)$, it is clear that any (normalized) eigenvector of $\Delta_{1}(k)$ must be of the form $\left(\alpha_{m k 1} \psi_{(m+1) l k}, \alpha_{m k 2} \psi_{(m-1) l k}, \alpha_{m k 3} \psi_{m l k}\right)$ for some $\alpha_{m k 1}, \alpha_{m k 2}, \alpha_{m k 3} \in \mathrm{C}$, with $\left|\alpha_{m k 1}\right|^{2}+\left|\alpha_{m k 2}\right|^{2}+\left|\alpha_{m k 3}\right|^{2}=1$ and 
$m=-1,0, \cdots$. Let us denote the corresponding eigenvalue by $\lambda(m, k)$. For fixed $m, \Delta_{1}(k)$ takes the form

$$
\Delta_{1}(m, k)=\left(\begin{array}{ccc}
2 k(m+1)+\frac{k^{2}}{c^{2}} & 0 & -c k^{1 / 2}(m+1)^{1 / 2} \\
0 & 2 k m+\frac{k^{2}}{c^{2}} & c k^{1 / 2} m^{1 / 2} \\
-c k^{1 / 2}(m+1)^{1 / 2} & c k^{1 / 2} m^{1 / 2} & k(2 m+1)+c^{2}+\frac{k^{2}}{c^{2}}
\end{array}\right) \text {. }
$$

For $m>0$, one eigenvalue of $\Delta_{1}(m, k)$ is given by $\lambda_{0}(m, k)=$ $(2 m+1) k+k^{2} / c^{2}$, with eigenvector

$$
\begin{aligned}
& \left(\alpha_{m k 1}, \alpha_{m k 2}, \alpha_{m k 3}\right) \\
& \quad=\left[(2 m+1) k+k^{2} / c^{2}\right)^{-1 / 2}\left(k^{1 / 2}(m+1)^{1 / 2}, k^{1 / 2} m^{1 / 2}, k / c\right] .
\end{aligned}
$$

This is in the image under $d$ of $\Lambda^{0}(G) \otimes C$ in $\Lambda^{1}(G) \otimes C$. The other eigenvalues turn out to be

$\lambda_{1,2}(m, k)=(2 m+1) k+k^{2} / c^{2}+c^{2} / 2 \pm c\left((2 m+1) k+k^{2} / c^{2}+c^{2} / 4\right)^{1 / 2}$

their eigenvectors will not be needed.

For $m=-1$, because $\psi_{(m-1) l k}$ and $\psi_{m l k}$ do not exist, there is only one eigenvalue, $\lambda(-l, k)=k^{2} / c^{2}$. For $m=0, \psi_{(m-1) l k}$ does not exist, and there are two eigenvalues, $\lambda_{0}(0, k)=k+k^{2} / c^{2}$ (which is in the image of $d)$ and $\lambda_{1}(0, k)=2 k+c^{2}+k^{2} / c^{2}$.

We can now compute the $k>0$ part of $\operatorname{tr} e^{-T \Delta_{1}}(0,0)$. As we already know $e^{-T \Delta_{0}}(0,0)$, it is enough to just consider the heat kernel of $\Delta_{1}$ acting on coclosed 1-forms; this is

$$
\begin{aligned}
\int_{0}^{\infty} \frac{d k}{2 \pi c} \sum_{l, m} e^{-T \lambda(m, k)}\left[\left|\alpha_{m k 1}\right|^{2}\left|\psi_{(m+1) l k}(0)\right|^{2}+\left|\alpha_{m k 2}\right|^{2}\left|\psi_{(m-1) l k}(0)\right|^{2}\right. & \left.+\left|\alpha_{m k 3}\right|^{2}\left|\psi_{m l k}(0)\right|^{2}\right] \\
4)= & \int_{0}^{\infty} \frac{d k}{2 \pi c} \frac{k}{2 \pi} \sum_{m} e^{-T \lambda(m, k)}\left[\left|\alpha_{m k 1}\right|^{2}+\left|\alpha_{m k 2}\right|^{2}+\left|\alpha_{m k 3}\right|^{2}\right] \\
= & \int_{0}^{\infty} \frac{d k}{2 \pi c} \frac{k}{2 \pi} \sum_{m} e^{-T \lambda(m, k)} .
\end{aligned}
$$


Substituting the expressions for the eigenvalues, we obtain

$$
\begin{array}{r}
\int_{0}^{\infty} \frac{d k}{2 \pi c} \frac{k}{2 \pi}\left\{\sum _ { m = 1 } ^ { \infty } \operatorname { e x p } \left(-T\left[(2 m+1) k+k^{2} / c^{2}+c^{2} / 2\right.\right.\right. \\
\left.\left. \pm c \sqrt{(2 m+1) k+k^{2} / c^{2}+c^{2} / 4}\right]\right) \\
\left.+e^{-T k^{2} / c^{2}}+e^{-T\left(2 k+k^{2} / c^{2}+c^{2}\right)}\right\} .
\end{array}
$$

One can check that the $k<0$ part is the same.

For the large $T$ asymptotics, the contributions of the last two terms of (135) are

$$
2 \int_{0}^{\infty} \frac{d k}{2 \pi c} \frac{k}{2 \pi} e^{-T k^{2} / c^{2}}=O\left(T^{-1}\right),
$$

which is that of the subspace $\mathscr{S}^{p}$ of Proposition 51, and

$$
2 \int_{0}^{\infty} \frac{d k}{2 \pi c} \frac{k}{2 \pi} e^{-T\left[2 k+c^{2}+k^{2} / c^{2}\right]}=o\left(T^{-1}\right) .
$$

Also

$$
\begin{aligned}
2 \int_{0}^{\infty} \frac{d k}{2 \pi c} \frac{k}{2 \pi} \sum_{m=1}^{\infty} & \exp \left(-T\left[(2 m+1) k+k^{2} / c^{2}+c^{2} / 2\right.\right. \\
& \left.\left.+c \sqrt{(2 m+1) k+k^{2} / c^{2}+c^{2} / 4}\right]\right)=o\left(T^{-1}\right),
\end{aligned}
$$

and so it only remains to look at

$$
\begin{aligned}
2 \int_{0}^{\infty} \frac{d k}{2 \pi c} \frac{k}{2 \pi} \sum_{m=1}^{\infty} \exp (-T & {\left[(2 m+1) k+k^{2} / c^{2}+c^{2} / 2\right.} \\
& \left.\left.-c \sqrt{(2 m+1) k+k^{2} / c^{2}+c^{2} / 4}\right]\right) .
\end{aligned}
$$

Changing variable to $u=\sqrt{(2 m+1) k+k^{2} / c^{2}+c^{2} / 4}-c / 2$, we find that (139) equals

$$
\frac{c}{2 \pi^{2}} \int_{0}^{\infty} d u \frac{u+c / 2}{l^{T u^{2}}} \sum_{m=1}^{\infty} \frac{\sqrt{u^{2}+c u+c^{2}\left(m+\frac{1}{2}\right)^{2}}-c\left(m+\frac{1}{2}\right)}{\sqrt{u^{2}+c u+c^{2}\left(m+\frac{1}{2}\right)^{2}}}
$$

Expanding in a Taylor's series yields that

$$
\sum_{m=1}^{\infty} \frac{\sqrt{u^{2}+c u+c^{2}(m+1 / 2)^{2}}-c(m+1 / 2)}{\sqrt{u^{2}+c u+c^{2}(m+1 / 2)^{2}}}=O(u) \quad \text { as } u \rightarrow 0,
$$

so that $(140)$ is $O\left(T^{-1}\right)$ as $T \rightarrow \infty$. 
Note. Some of the results of $\S \S I I I$ and IV have been independently obtained by V. Mathai [34]. M. Gromov and M. Shubin have shown that $\alpha_{p}(M)$ is a homotopy invariant [27].

\section{Acknowledgments}

The author wishes to thank M. Christ and M. Gromov for helpful suggestions, and W. Lück, S. Novikov, and M. Shubin for explanations of their work. The author also thanks M. Berger and the Institut des Hautes Études Scientifiques for their hospitality while this work was performed, the Sloan Foundation for partial support, and the referee for his/her suggestions.

\section{References}

[1] M. Atiyah, Elliptic operators, discrete groups and von Neumann algebras, Astérisque 32 (1976) 43-72.

[2] E. Bonan, Sur l'algèbre extérieure d'une variété presque Hermitienne quaternionique, C. R. Acad. Sci. Paris Sér. I Math. 295 (1982) 115-118.

[3] A. Borel \& N. Wallach, Continuous cohomology, discrete subgroups and representations of reductive groups, Annals of Math. Studies, No. 94, Princeton University Press, Princeton, NJ, 1980.

[4] R. Brooks, The fundamental group and the spectrum of the Laplacian, Comment. Math. Helv. 56 (1981) 581-598.

[5] R. Cahn, P. Gilkey \& J. Wolf, Heat equation, proportionality principle and volume of fundamental domains, Differential Geometry and Relativity, Math. Phys. and Appl. Math., Vol. 3, Reidel, Dordrecht, 1976, 43-54.

[6] A. Carey \& V. Mathai, $L^{2}$ acyclicity and $L^{2}$-torsion invariants, preprint, 1989.

[7] J. Cheeger, Analytic torsion and the heat equation, Ann. of Math. (2) 109 (1979) 259322.

[8] J. Cheeger \& M. Gromov, On the characteristic numbers of complete Manifolds of bounded curvature and finite volume, Differential Geometry and Complex Analysis, Rauch Memorial Volume (I. Chavel and H. Farkas, ed.), Springer, Berlin, 1985.

[9] __ Bounds on the von Neumann dimension of $L^{2}$ cohomology and the Gauss-Bonnet theorem for open manifolds, J. Differential Geometry 21 (1985) 1-34.

[10] $\ldots, L^{2}$ cohomology and group cohomology, Topology 25 (1986) 189-215.

[11] J. Cheeger, M. Gromov \& M. Taylor, Finite propagation speed, kernel estimates for functions of the Laplace operator and the geometry of complete Riemannian manifolds, J. Differential Geometry 17 (1982) 15-53.

[12] J. Cheeger \& S. T. Yau, A lower bound for the heat kernel, Comm. Pure Appl. Math. 34 (1981) 465-480.

[13] G. De Cecco \& G. Palmieri, Length of curves on lip manifolds, Universita Degli Studi di Lecce preprint, 1989.

[14] J. Dixmier, Von Neumann algebras, North-Holland, Amsterdam, 1981.

[15] J. Dodziuk, De Rham-Hodge theory for $L^{2}$ cohomology of infinite coverings, Topology 16 (1977) 157-165. 
[16] H. Donnelly, The differential form spectrum of hyperbolic space, Manuscripta Math. 33 (1980/81) 365-385.

[17] H. Donnelly \& F. Xavier, On the differential form spectrum of negatively curved Riemannian manifolds, Amer. J. Math. 106 (1984) 169-185.

[18] D. Efremov \& M. Shubin, Spectrum distribution function and variational principle for automorphic operators on hyperbolic space, Seminaire EDP 1988/1989, Exposé VIII, Ecole Polytechnique, Paris.

[19] D. Fried, Analytic torsion and closed geodesics on hyperbolic manifolds, Invent. Math. 84 (1986) 523-540.

[20] __ Lefschetz formulas for flows, Contemp. Math. 58 III (1987) 19-69.

[21] B. Gaveau, Principe de moindre action, propagation de la chaleur et estimées sous elliptiques sur certains groupes nilpotents, Acta. Math. 139 (1977) 95-153.

[22] P. Griffiths \& J. Morgan, Rational homotopy theory and differential forms, Birkhäuser, Boston, 1981.

[23] M. Gromov, Structures métriques pour les variétés Riemanniennes, Cedic-FernandNathan, Paris, 1981.

[24] __ Groups of polynomial growth and expanding maps, Inst. Hautes Études Sci. Publ. Math. 53 (1981) 53-73.

[25] __, Volume and bounded cohomology, Inst. Hautes Études Sci. Publ. Math. 56 (1982) 5-99.

[26] Kähler hyperbolicity and $L^{2}$ Hodge theory, preprint IHES/M/89/29, 1989.

[27] M. Gromov \& M. Shubin, Von Neumann spectra near zero, preprint, 1990.

[28] M. Hilsum, Signature operator on Lipschitz manifolds and unbounded Kasparov bimodules, Operator Algebras and the Connections with Topology and Ergodic Theory, Lecture Notes in Math., Vol. 1132, Springer, Berlin, 1985.

[29] __ Fonctorialié en $K$-théorie bivariante pour les variétés Lipschitziennes, $K$-Theory, to appear.

[30] J. Lott, The eta function and some new anomalies, Phys. Lett. B 145 (1984) 179-180.

[31] W. Lück \& M. Rothenberg, PL torsion and the $K$ theory of von Neumann algebras, to appear.

[32] J. Luukkainen \& J. Väisälä, Elements of Lipschitz topology, Ann. Acad. Sci. Fenn. Ser. A I Math. 3 (1977) 85.

[33] B. Malgrange, Intégrales asymptotiques et monodromie, Ann. Sci. École Norm. Sup. (4) 7 (1974) 405-430.

[34] V. Mathai, $L^{2}$ analytic torsion, preprint, 1990.

[35] R. Miatello, On the Plancherel measure for linear lie groups of rank one, Manuscripta Math. 29 (1979) 249-276.

[36] __ The Minakshisundaram-Pleijel coefficients for the vector-valued heat kernel on compact locally symmetric spaces of negative curvature, Trans. Amer. Math. Soc. 260 (1980) 1-33.

[37] J. Milnor, A note on curvature and fundamental group, J. Differential Geometry 2 (1968) 1-7.

[38] __ Infinite cyclic coverings, Conference on the Topology of Manifolds, Michigan State University, East Lansing 1967, Prindle, Weber and Schmidt, Boston, 1968, 115-133.

[39] H. Moscovici \& R. Stanton, R-torsion and zeta functions for locally symmetric manifolds, Ohio State preprint, 1989.

[40] W. Müller, Analytic torsion and the R-torsion of Riemannian manifolds, Advances in Math. 28 (1978) 233-305.

[41] S. Novikov \& M. Shubin, Morse inequalities and von Neumann invariants of non-simplyconnected manifolds, Uspekhi Mat. Nauk 41 (1986) No. 5, 222-223. (Russian)

[42] D. Ray \& I. Singer, $R$-torsion and the laplacian on Riemannian manifolds, Advances in Math. 7 (1971) 145-210. 
[43] M. Reed \& B. Simon, Methods of mathematical physics IV: Analysis of operators, Academic Press, New York, 1978.

[44] P. Scott, The geometries of 3-manifolds, Bull. London Math. Soc. 15 (1983) 401-487.

[45] M. Shubin, Weyl theorem for the Schrödinger operator with almost periodic potential, Vestnik Moscov. Univ. Ser. I Mat. Mekh. 31 (1976) No. 2, 84-88. (Russian)

[46] A. Shvarts, The partition function of a degenerate functional, Comm. Math. Phys. 67 (1979) 1-16.

[47] D. Sullivan, Hyperbolic geometry and homeomorphisms, Geometric Topology, Proc. Georgia Top. Conf., Athens, GA, 1977, Academic Press, New York, 1979, 543-555.

[48] N. Teleman, The index of signature operators on Lipschitz manifolds, Inst. Hautes Études Sci. Publ. Math. 58 (1983) 39-78.

[49] N. Varapoulos, Random walks and Brownian motion on manifolds, Sympos. Math., Vol. 29, Academic Press, New York, 1988, 97-109.

[50] S. Vishik, Analogs of Selberg's $\zeta$-function, Functional Anal. Appl. 9 (1975) 256-257.

[51] D. Vogan \& G. Zuckeman, Unitary representations with nonzero cohomology, Compositio Math. 53 (1984) 51-90.

[52] T. Yoshida, The $\eta$ invariant of hyperbolic 3-manifolds, Invent. Math. 81 (1985) 473514.

UNIVERSITY OF MICHIGAN 